\title{
MedienPädagogik
}

Zeitschrift für Theorie und Praxis der Medienbildung

Themenheft Nr. 42: Optimierung in der Medienpädagogik.

Forschungsperspektiven im Anschluss an den 27. Kongress der DGfE

Herausgegeben von Patrick Bettinger, Klaus Rummler und Karsten D. Wolf

\section{Leistungsoptimierung von Schülerinnen und Schülern durch schulbezogene Erklärvideonutzung auf YouTube}

\section{Entschulungsstrategie oder Selbsthilfe?}

Karsten D. Wolf ${ }^{1}$ D, Ilona Andrea Cwielong ${ }^{2}$ iD, Sven Kommer ${ }^{2}$ und Katrin Ellen Klieme ${ }^{1}$ (D)

${ }^{1}$ Universität Bremen

${ }^{2}$ RWTH Aachen University

\section{Zusammenfassung}

Die schulbezogene Nutzung von Erklärvideos insbesondere auf YouTube hat sich zunehmend im Medienrepertoire von Schülerinnen und Schülern etabliert. Gegenstand des Beitrags ist die Beschreibung und Analyse der ausserschulischen Nutzung von Erklärvideos in verschiedenen Schulfächern als Form schulbezogener Leistungsoptimierungsstrategien. Von Interesse ist, welche Fächer geschaut werden, welche Nutzungsanlässe und motivationalen Gründe es zur Rezeption gibt sowie welche Lernstrategien dabei genutzt werden. Ein Schwerpunkt liegt dabei auf der Analyse, wie sich die schulbezogene Erklärvideonutzung von Schülerinnen und Schülern auf unterschiedlichen Notenniveaus unterscheiden. Die Datenbasis bildet eine standardisierte Befragung $(n=1.392)$ von Schülerinnen und Schülern der 8. bis 13. Klasse in den Regionen Bremen und Aachen an allgemeinbildenden Schulen. Als Ergebnis zeigt sich, dass insbesondere Erklärvideos für die Schulfächer Mathematik, Geschichte sowie Biologie rezipiert werden. Schülerinnen und Schüler mit schlechteren Noten schauen überproportional viele Erklärvideos in den Nachhilfe relevanten Fächern Mathematik, Deutsch sowie Englisch und geben mehr fremdbestimmte Gründe für das Schauen von Erklärvideos an. Schülerinnen und Schüler mit besseren Noten nutzen Erklärvideos eher selbstbestimmt und nutzen verstärkt verstehensorientierte Lernstrategien. Abschliessend wird herausgearbeitet, dass die Nutzung durch die Schülerinnen und Schüler als eine leistungs- bzw. effizienzoptimierende Selbsthilfestrategie zu verstehen ist. Obwohl die Bereitstellung und breite Rezeption von Erklärvideos ein Indiz für eine zunehmende Entschulungsstrategie darstellt, zeigt die Analyse, dass das curriculare Hegemonial der Schule in Bezug auf die Inhalte schulbezogener Erklärvideos weiter besteht. 


\title{
Students' Optimization of their School Performance by Using Explainer Videos on YouTube. Deschooling Strategy or Self-Help?
}

\begin{abstract}
School-related use of explainer videos, especially on YouTube, has increasingly established itself in the media repertoire of students. This article describes and analyzes the use of explainer videos outside of school as a form of school-related performance optimization strategies in various school subjects. The investigation aims to clarify which subjects are watched, which occasions for the use exist, which reasons for the reception are given and which learning strategies are used. One focus is on the analysis of how school-related explanatory video use differs among students at different grade levels. The data basis is a standardized survey $(n=1.392)$ of $8^{\text {th }}$ to $13^{\text {th }}$ grade students at general education schools in the regions of Bremen and Aachen. The results show that explanatory videos are watched particularly frequently for the school subjects mathematics, history and biology. Students with poorer grades watch a disproportionately high number of videos in math, German and English. They also give more externally determined reasons for watching explainer videos. Students with better grades are more likely to use explanatory videos in a selfdetermined way and use more comprehension-oriented learning strategies. In conclusion, we find that students' use of explanatory videos should be understood as a performanceor efficiency-optimizing self-help strategy. Although the provision and widespread reception of explainer videos is an indication of an increasing deschooling strategy, the analysis shows that the curricular hegemony of the school continues to exist with regard to the content of school-related explainer videos.
\end{abstract}

\section{Präambel in Zeiten des Corona-Lockdowns}

In Zeiten des ersten Corona-Lockdowns fehlte es nicht an Optimierungswünschen und -appellen an das deutsche Schulsystem, den Ausnahmezustand mittels digitaler Tools in den Griff zu bekommen. So forderte die Bundesbildungsministerin Anja Karliczek im Frühjahr 2020:

«Nach den Ferien muss überall ein strukturierter Unterricht angeboten werden - und zwar so, dass möglichst ein volles Schulprogramm gewährleistet ist. Wie auch immer.» (Rheinische Post, 30.05.2020)

Der sich aus dieser Situation ergebende Handlungs(spiel)raum im traditionell eher medienskeptischen deutschen Schulsystem (Kommer 2010, 2016; Wolf 2017) war nicht nur vollkommen neu, sondern wurde je nach Habitus der Lehrpersonen als Chance oder kaum zu bewältigende Herausforderung empfunden. Dies nicht zuletzt vor dem Hintergrund, dass Ministerien, Schulleitungen, aber auch die Schulträger 
überwiegend keine klaren Konzepte oder Vorgaben für eine digitale Beschulung vorlegten und die Infrastruktur oft mangelhaft war. Letztendlich war hier viel Improvisationstalent gefordert. Dabei konnte eine Gruppe von digital-affinen Lehrpersonen gemeinsam prototypische Konzepte für den digital unterstützten Unterricht entwickeln (Kantereit 2020).

Erklärvideos spielten (und spielen) in dem digitalen Notfall-Unterricht eine wichtige Rolle als Ersatz für fehlende Präsenzzeiten und ausfallenden Unterricht. Sie werden teils von den Lehrpersonen für die Schülerinnen und Schüler ausgewählt und bereitgestellt, teils von den Lernenden selbst aktiv gesucht und genutzt (Wolf und Kulgemeyer 2021). Die Anleitung oder Durchführung didaktisch-methodischer Prozesse wie das Erklären, Erarbeiten, Explorieren oder Üben liegen damit nicht mehr alleine in der Hand der professionellen Lehrpersonen, sondern werden an die Lernenden bzw. die Produzierenden der Erklärvideos delegiert. Schülerinnen und Schüler müssen - teils mit Unterstützung ihrer Eltern - ihre Lernprozesse unter Einbezug digitaler Medien selbst organisieren. Dieser Beitrag beschreibt auf Basis von Erhebungsdaten, welche direkt vor dem ersten Corona-Lockdown Anfang 2020 erhoben wurden, die Ausgangslage für anstehende weiterführende Untersuchungen der Digitalisierungsprozesse in den Schuljahren 2020 bis 2021.

\section{Problemstellung}

Als Ivan Illich 1971 die «Entschulung der Gesellschaft» forderte, waren ein ubiquitäres Internet und Pandemie induzierte Lockdowns der Schule noch sehr weit weg. In seiner auf Gesellschaftsveränderung zielenden Streitschrift entwickelte er die Utopie eines ‘wirklichen> Lernens jenseits eines auf (falsche) Anpassung und subtile Unterdrückung orientierten Schulsystems.

«Die radikalste Alternative zur Schule wäre ein Netzwerk oder ein Service, der jedermann [sic!] die gleiche Gelegenheit bietet, seine jeweiligen Anliegen mit anderen zu teilen, welche dieselben Anliegen haben» (Illich 1971, 40).

Was damals als fernab aller Realisierungsmöglichkeiten erschien, ist heute längst Alltag (auch wenn der revolutionäre Anspruch zunächst auf der Strecke geblieben ist): Auf (hochkommerziellen) Portalen wie YouTube hat sich mit dem Genre der Erklärvideos und Tutorials vom Bildungssystem weitgehend unreflektiert die Utopie von Illich und anderen realisiert - jede und jeder kann hier Lehr-/Lernangebote ausbringen und/oder nutzen, ohne auch nur am Rande mit dem formalen Bildungssystem in Kontakt zu kommen (Wolf 2015a; Ito et al. 2018).

Schon vor den Schul-Lockdowns in den Jahren 2020 und 2021 ist nicht nur ein grosses Angebot, sondern auch eine intensive Nutzung von Erklärvideos zu beobachten: Laut der JIM-Studie 2019 gaben 18 \% der befragten 12- bis 19-jährigen Jugendlichen 
an, täglich oder mehrmals pro Woche «Erklärvideos für Schule(Ausbildung)» anzusehen (Feierabend u. a. 2020). Die Studie «Jugend/YouTube/Kulturelle Bildung. Horizont 2019» dokumentiert ebenfalls die grosse Bedeutung von Erklärvideos für das schulbezogene Lernen: YouTube-Videos zu Schulthemen sind für $47 \%$ der 12 - bis 19-jährigen Jugendlichen wichtig bzw. sehr wichtig, insbesondere um Inhalte nachzuarbeiten, die sie in der Schule nicht verstanden haben (Rat für kulturelle Bildung 2019, 55).

Für das von Schulschliessungen und Online-Unterricht betroffene Jahr 2020 unterscheiden sich die Daten der jährlich durchgeführten JIM-Studie jedoch nicht substantiell von den Vorjahreswerten: $21 \%$ der befragten 12- bis 19-jährigen Jugendlichen (+3 Prozentpunkte) geben an, täglich oder mehrmals pro Woche «Erklärvideos/Tutorials für Schule/Ausbildung» anzusehen (JIM-Studie 2020: Feierabend, Rathgeb, und Reutter 2020, 47; Basis: Befragte, die zumindest seltener YouTube nutzen, $\mathrm{n}=1.194)$. Eine sprunghafte Intensivierung der Nutzung von Erklärvideos lässt sich aus den vorliegenden, hochaggregierten Daten der JIM-Studie nicht herauslesen. In der JIMplus 2020 Corona-Zusatzuntersuchung zur ersten Corona induzierten Schulschliessung berichten ohne weitere Angabe des Nutzungsumfanges $45 \%$ der befragten 12- bis 19-jährigen Schülerinnen und Schüler, «Tutorials im Internet» zu nutzen («Wer hilft dir beim Lernen?») (Rathgeb 2020, 8). Nur das Lernen mit Freunden über Chat (50\%) und die Hilfe durch Eltern (43\%) wird ähnlich häufig angegeben. Bezüglich der genutzten medialen Lernangebote liegt die Nutzung von YouTube (83\%) noch vor Wikipedia $(58 \%)^{1}$. Öffentlich-rechtliche Angebote wie Dokus (Dokumentationen) und Wissenssendungen im Fernsehen/Mediatheken bzw. Schulsendungen werden dagegen nur von ca. einem Viertel der Befragten genutzt ${ }^{2}$ (Rathgeb 2020,11). Insgesamt kann man davon ausgehen, dass die schulbezogene Nutzung von Erklärvideos durch Jugendliche auch vor dem Lockdown bereits fest in deren Medienrepertoires verankert war.

Trotz der breiten Nutzung durch Schülerinnen und Schüler liegen aus den bisherigen vorliegenden empirischen Studien nur wenig differenzierte Daten zu Erklärvideos vor. Im Rahmen eines gemeinsamen Forschungsprojektes «Digitale außerschulische Lern- und Bildungspraxen Jugendlicher (DAB-J)» $»^{3}$ der RWTH Aachen und der Universität Bremen sowie im Forschungsprojekt «Lernen in einer digitalen Welt - schulbezogene Mediennutzung von Schülerinnen und Schülern ${ }^{4}$ an der Universität Bremen

1 Hier ist nicht vollständig klar, warum die Nutzung von YouTube zwar von $83 \%$ der befragten Jugendlichen berichtet wird, bei der Frage nach der Hilfe beim Lernen «Tutorials im Internet» aber nur mit $45 \%$ angegeben wird. Gegebenenfalls ist der Begriff «Tutorials» zu eng gewählt, da er eine klare Schritt-für-SchrittAnleitung erwarten lässt, während YouTube als eine Art audio-visueller Enzyklopädie auch Videoformen wie Dokumentationen, Wissens- oder Erklärvideos i.e.S. umfasst (Wolf 2015a, b).

2 Hier zeigt sich ein Reichweiten- und Formatproblem der öffentlich-rechtlichen Mediatheken im Vergleich zu YouTube an. Weder sind die Mediatheken im Medienrepertoire der Jugendlichen verankert, noch entsprechen die Formate den von YouTube geprägten Sehgewohnheiten.

3 BMBF Förderkennzeichen 01JD1804A und 01JD1804B.

4 Gefördert durch die Mercartor Stiftung. 
sollen deshalb weitergehende Fragen zur Erklärvideonutzung von Schülerinnen und Schülern empirisch geklärt werden. Von Interesse ist dabei, für welche Fächer Erklärvideos genutzt werden, welche Nutzungsanlässe und motivationale Gründe es zur Rezeption gibt sowie welche Lernstrategien dabei genutzt werden. Ein Schwerpunkt liegt dabei auf der Analyse, wie sich die schulbezogene Erklärvideonutzung von Schülerinnen und Schülern auf unterschiedlichen Notenniveaus unterscheiden. Abschliessend wird die Frage diskutiert, ob die Nutzung von Erklärvideos eher eine Selbsthilfestrategie der Schülerinnen und Schüler darstellt oder ob die Bereitstellung von Erklärvideos auf kommerziellen Plattformen wie YouTube oder Sofatutor als Vorboten einer zunehmenden Entschulungsstrategie interpretiert werden können.

\section{Schulische Leistungsoptimierung mit Erklärvideos?}

In der Wirtschaftstheorie beschreibt das «Ökonomische Prinzip» das überwiegend rationale Verhalten von Menschen, das Verhältnis von Mitteleinsatz (Input bzw. Aufwand) und dem Ergebnis (Output, Ertrag oder Ziel) in einem gewissen Masse zu optimieren (Zydorek 2017; zur Kritik des «Rational Choice» Modell vgl. Müller 2012). Bezogen auf das schulische Bildungssystem ergibt sich die Problematik, die relevanten und vielfältigen Gestaltungsparameter sowie den Output oder Ertrag zu quantifizieren, um überhaupt eine Modellierung des Wirkgefüges optimieren zu können (Ricken 2020, 33). Aus Sicht der Schülerinnen und Schüler mag dies zu mindestens bezüglich des Outputs nicht sonderlich schwierig erscheinen. Spätestens in der Sekundarstufe wird der Ertrag schulischer Leistungen in Form von Ziffernnoten dokumentiert, welche als Leistungsindikatoren zwar von den Eltern und Lehrpersonen - je nach Schulstufe, Schulform und eigenem Bildungsniveau unterschiedlich - zum Teil kritisch wahrgenommen, von den Schülerinnen und Schülern der Sekundarstufe aber weitgehend akzeptiert werden (Jachmann 2003).

Bezüglich der Abschätzung des Inputs bzw. des Lernaufwands erscheint dies ungleich komplexer und ist schwieriger zu operationalisieren. Aus Sicht einzelner Schülerinnen und Schüler bieten sich - neben der mehr oder weniger aktiven Teilhabe am für alle verpflichtenden Unterricht - verschiedene Formen und Qualitäten des Ressourceneinsatzes an, wie insbesondere die eingesetzte aktive Lernzeit (Helmke 2021), der Einsatz von Lernstrategien (Donker et al. 2014), Lernunterstützung durch Peers, Familienmitglieder oder Nachhilfelehrpersonen (Wagner et al. 2005; Jürgens 2008) sowie die Beschaffung und Nutzung von Lernmitteln (Herzig 2017; Zwingenberger 2009) wie z. B. Übungshefte oder eben auch digitale Ressourcen wie die hier näher betrachteten Erklärvideos.

Das Ökonomische Prinzip lässt sich im Kontext schulischer Leistungsoptimierung wie folgt in drei mögliche Ausprägungen formulieren: 
1. Das Minimalprinzip lautet, eine angestrebte Note (vorgegebener Output; Beispiele: «In allen Hauptfächern eine 2!», «Keine 5 im Zeugnis!» oder «Einen Abiturdurchschnitt von 1,2!») mit einem möglichst geringen Lernaufwand (minimaler Input) zu erreichen.

2. Das Maximalprinzip geht davon aus, mit einem gegebenen Lern- bzw. Ressourcenaufwand (Beispiele: «Jeden Wochentag 2 Stunden für die Schule lernen», «Für die Klassenarbeit am Freitag mit der großen Schwester 4 Stunden lernen», «50€ für Nachhilfe und Lernmittel ausgeben») eine bestmögliche Note (maximaler Output) zu erreichen.

3. Das Optimumprinzip schliesslich vermittelt zwischen beiden Prinzipien, indem das Verhältnis zwischen Mitteleinsatz und Output optimiert wird. So könnte z. B. die Anforderung «In allen Hauptfächern eine 2!» bei einem speziellen Fach zu einem unrealistisch hohen zeitlichen Aufwand führen, so dass eine Mischung aus Minimalprinzip («Nur so viel lernen, dass ich in allen Hauptfächern bis auf Englisch eine 2 habe») mit dem Maximalprinzip («Jeden Tag konzentriert eine Stunde Englisch lernen, um von der aktuellen 5 in Englisch auf eine bestmögliche Note zu kommen») kombiniert wird.

Inwieweit Schülerinnen und Schüler Noten nur als Nebenbedingung der schulbezogenen Output-Optimierung verstehen, da sie als relativ subjektiv und wenig bedeutsam für den eigenen Lernerfolg verstanden werden, ist empirisch nicht geklärt. So könnte (ggf. auch nur für einzelne Fächer) das erreichte Verständnisniveau, die Beantwortung subjektiv interessanter Fragen oder der wahrgenommene Kompetenzgewinn im Mittelpunkt der eigenen Lernbemühungen stehen und als optimierungsleitendes Output-Mass dienen. Die hohe Akzeptanz von Ziffernzensuren durch die Schülerinnen und Schüler in der Sekundarstufe (Jachmann 2003) lassen jedoch vermuten, dass die Optimierung von Noten beim schulischen Lernen eine zentrale Rolle spielen.

Der Einsatz von Erklärvideos ist für alle vorgestellten Optimierungsprinzipien denkbar. Die Lernzeit kann minimiert werden, wenn z. B. mit Erklärvideos ein Thema der Klassenarbeit schneller erarbeitet werden kann als mit anderen Lernressourcen wie dem Schulbuch oder eigenen Aufzeichnungen. Die Note eines Referates kann maximiert werden, wenn z. B. das Schauen verschiedener Erklärvideos in der gegebenen Vorbereitungszeit eine bessere Übersicht, griffigere Argumente, verständlichere Erklärungen oder mehr anschauliche Beispiele vermittelt als das Recherchieren in der Schulbibliothek oder das Lesen eines Textes in derselben Zeit.

Zur weiteren Systematisierung des möglichen Beitrages von Erklärvideos zur Leistungsoptimierung von Schülerinnen und Schülern greifen wir auf das AktiotopModel der Hochbegabung (Ziegler et al. 2017) zurück (siehe Tab. 1). Als Aktiotop versteht Ziegler die Gesamtheit des handelnden Individuums und seines materiellen, 
sozialen und informativen Umfelds (Ziegler, Vialle, und Wimmer 2013, 3). Ziegler differenziert die von Bourdieu beschriebenen Kapitalsorten (ökonomisches, kulturelles, soziales Kapital; Bourdieu 1983) in Bezug auf das Lernen in exogene Ressourcen (Bildungskapital) und endogene Ressourcen (Lernkapital). Tabelle 1 listet die von Ziegler benannten Kapitalsorten auf.

\begin{tabular}{|c|c|c|c|}
\hline \multicolumn{2}{|c|}{ Bildungskapital: exogene Lernressourcen } & \multicolumn{2}{|c|}{ Lernkapital: endogene Lernressourcen } \\
\hline Sorten & $\begin{array}{l}\text { Bsp. für Potentiale } \\
\text { durch Erklärvideobe- } \\
\text { reitstellung }\end{array}$ & Sorten & $\begin{array}{l}\text { Bsp. für Impulse } \\
\text { durch Erklärvideo- } \\
\text { nutzung }\end{array}$ \\
\hline $\begin{array}{l}\text { Ökonomisches } \\
\text { Bildungskapital }\end{array}$ & $\begin{array}{l}\text { Kostenfreier Zugriff } \\
\text { auf ansonsten kosten- } \\
\text { pflichtiger Nachhilfe- } \\
\text { angebote }\end{array}$ & $\begin{array}{l}\text { Organismisches } \\
\text { Lernkapital }\end{array}$ & $\begin{array}{l}\text { Informationen über } \\
\text { und Wege zur Verbes- } \\
\text { serung von Körper } \\
\text { und Geist durch spezi- } \\
\text { elle Tutorials }\end{array}$ \\
\hline $\begin{array}{l}\text { Infrastrukturelles } \\
\text { Bildungskapital }\end{array}$ & $\begin{array}{l}\text { Vereinfachter Zu- } \\
\text { gang zu Erklär- und } \\
\text { Übungsressourcen } \\
\text { über Smartphone }\end{array}$ & $\begin{array}{l}\text { Aktionales } \\
\text { Lernkapital }\end{array}$ & $\begin{array}{l}\text { Übungsanleitungen } \\
\text { zum Erwerb von Fer- } \\
\text { tigkeiten und Fähig- } \\
\text { keiten (Tutorials) }\end{array}$ \\
\hline $\begin{array}{l}\text { Kulturelles } \\
\text { Bildungskapital }\end{array}$ & $\begin{array}{l}\text { Exposition mit ande- } \\
\text { ren Wertesystemen, } \\
\text { Denkmustern und } \\
\text { Modellen durch die } \\
\text { Erklärvideo-Produzie- } \\
\text { renden }\end{array}$ & $\begin{array}{l}\text { Telisches } \\
\text { Lernkapital }\end{array}$ & $\begin{array}{l}\text { Ratschläge und Im- } \\
\text { pulse zur Entwicklung } \\
\text { von Begeisterung für } \\
\text { ein Thema; Orientie- } \\
\text { rung und Unterstüt- } \\
\text { zung beim Setzen } \\
\text { und Beibehalten von } \\
\text { Zielen }\end{array}$ \\
\hline $\begin{array}{l}\text { Soziales } \\
\text { Bildungskapital }\end{array}$ & $\begin{array}{l}\text { Feedback und Unter- } \\
\text { stützung durch Kom- } \\
\text { mentare der Peers } \\
\text { sowie Produzierenden }\end{array}$ & $\begin{array}{l}\text { Attentatives } \\
\text { Lernkapital }\end{array}$ & $\begin{array}{l}\text { Fokussierung der } \\
\text { Lernzeit auf tatsäch- } \\
\text { liches Üben und } \\
\text { zielgerichtete Be- } \\
\text { schäftigung mit einem } \\
\text { Thema }\end{array}$ \\
\hline $\begin{array}{l}\text { Didaktisches } \\
\text { Bildungskapital }\end{array}$ & $\begin{array}{l}\text { Zugang zu videoba- } \\
\text { sierten Erklärungen } \\
\text { und didaktischen Trai- } \\
\text { ningsprogrammen }\end{array}$ & $\begin{array}{l}\text { Episodisches } \\
\text { Lernkapital }\end{array}$ & $\begin{array}{l}\text { Lernen von Hand- } \\
\text { lungsmustern am Mo- } \\
\text { dell der Erklärvideo- } \\
\text { Produzierenden in } \\
\text { spezifischen Anwen- } \\
\text { dungssituationen }\end{array}$ \\
\hline $\begin{array}{l}\text { Siehe zur Definitio } \\
\text { Hauptschülern) }\end{array}$ & ssourcen Ziegler et & 12 (Bildungs & kapitalarmut bei \\
\hline
\end{tabular}

Tab. 1.: Zusammenfassung potentieller positiver Effekte der Bereitstellung bzw. Nutzung von Erklärvideos auf das Bildungs- und Lernkapital von Schülerinnen und Schülern nach Ziegler (eigene Darstellung). 
Wenig überraschend ist der Zugang zu Bildungs- und Lernkapital im Schulsystem bisher ungleich verteilt. In einer explorativen Studie, in der 157 Lehrpersonen zum Kapitalzugang ihrer Schülerinnen und Schüler an verschiedenen Schulformen befragt wurden, werden für Schülerinnen und Schüler an Hauptschulen ein geringeres Bildungs- und Lernkapital berichtet als für Schülerinnen und Schüler der Realschule sowie Gymnasiastinnen und Gymnasiasten (Ziegler et al. 2012).

Ungeklärt ist bisher, ob Erklärvideos zur Verbesserung der Ausstattung von Schülerinnen und Schülern mit Bildungs- und Lernkapital beitragen können (siehe auch Tab. 1). Bezogen auf das Bildungskapital nach Ziegler belegen die oben referierten Studien, dass die Mehrheit der Schülerinnen und Schüler Erklärvideos in ihre Medienrepertoires integriert haben (siehe Kapitel 1). Zu klären ist, ob diese als exogene Lernressourcen für eine Verbesserung des Bildungskapitals dienen können. So erlaubt die Bereitstellung von Erklärvideos auf YouTube einen kostenfreien Zugang zu ansonsten kostenpflichtigen Nachhilfeangebote. Sie könnten dadurch die Bedeutung des verfügbaren ökonomischen Bildungskapitals verringern, indem finanzielle Ressourcen für die Bezahlung z. B. von Nachhilfe weniger wichtig sind für die individuelle Lernunterstützung von Schülerinnen und Schülern. Durch das Angebot von Erklärungen und Übungsanleitungen erhöhen sie das didaktische Bildungskapital. Durch die Bereitstellung von Erklärvideos auf YouTube über Smartphones wird infrastrukturelles Bildungskapital aufgebaut, da z. B. nicht erst eine Bibliothek aufgesucht werden muss, um Bildungsressourcen zu nutzen. Erklärvideos können auch mit anderen bildungsorientierten Wertesystemen, Denkmustern und Modellen der Erklärenden bzw. der Erklärvideoproduzierenden vertraut machen und somit das kulturelle Bildungskapital der Schülerinnen und Schüler positiv verändern. Feedback und Unterstützung durch Kommentare der Peers sowie der Produzierenden erweitern idealerweise das soziale Bildungskapital.

Auch das Lernkapital (endogene Lernressourcen) kann durch Erklärvideos möglicherweise erweitert werden. Insbesondere in Tutorials können die konkreten Übungsanleitungen zum Erwerb von Fertigkeiten und Fähigkeiten mittelfristig das aktionale Lernkapital vergrössern und über eine Fokussierung der Lernzeit auf tatsächliches Üben und zielgerichtete Beschäftigung mit einem Thema schrittweise das attentative Lernkapital erweitern. Anhand der Handlungsmuster der Erklärvideoproduzierenden in spezifischen Anwendungssituationen kann durch Modelllernen das episodische Lernkapital erhöht werden. Meta-Kommentare der Erklärvideoproduzierenden in Form von Ratschlägen und Impulsen zur Entwicklung von Begeisterung für ein Thema sowie zur Orientierung und Unterstützung beim Setzen und Beibehalten von Zielen verstärken das telische Lernkapital. Schliesslich finden sich auf YouTube eine Vielzahl von Erklärvideos zur Optimierung der körperlichen und mentalen Gesundheit (organismisches Lernkapital). 
Erklärvideos könnten also einen gewissen Beitrag zur Bildungsgerechtigkeit leisten, indem sie Zugänge zu Lern- und Bildungskapital eröffnen. Allerdings ist in der Forschung zum Digital Divide bereits länger bekannt, dass das Angebot digitaler Ressourcen nicht automatisch Nutzung und positive Wirkung sichern (Van Deursen et al. 2015; Van Dijk 2020). Insbesondere die Such- und Empfehlungsalgorithmen von YouTube lassen einen Zero-Level Divide (Verständig, Klein, und Iske 2016) vermuten: die eigene Nutzungshistorie beeinflusst in hohem Masse die angebotenen Videos, so dass trotz eines scheinbar identischen Zuganges zu YouTube durchaus unterschiedliche lernunterstützende oder ablenkende Inhalte gefunden werden können.

Welche differentiellen Effekte Erklärvideos tatsächlich auf das Bildungs- und Lernkapital haben bzw. ob die einzelnen Kapitalsorten entscheidende Moderatoren für die erfolgreiche Nutzung von Erklärvideos sind, ist bisher allerdings weitgehend ungeklärt und stellt ein umfängliches Forschungsdesiderat dar.

\section{Empirische Fragestellungen}

Als erste Annäherung an die Frage, wie Schülerinnen und Schüler Erklärvideos für die schulische Leistungsoptimierung nutzen, soll in dieser Studie analysiert werden, wie sich die Nutzung von schulbezogenen Erklärvideos zwischen Schülerinnen und Schülern auf verschiedenen Leistungsniveaus unterscheiden. Dabei werden zwei Aspekte näher analysiert:

1. Nutzung nach Fächern und Schulleistung: Zunächst soll die Nutzung von Erklärvideos genauer beschrieben werden. Für welche Schulfächer werden Erklärvideos besonders häufig rezipiert und gibt es dabei Unterschiede zwischen Schülerinnen und Schüler auf verschiedenen Notenniveaus? Gibt es einen Zusammenhang zwischen Nutzungsintensität und der formalen Bedeutung («versetzungsrelevante Fächer») der Fächer? Oder findet sich nur im Umfeld nachhilfeintensiver Fächer eine stärkere Rezeption?

2. Formen und Strategien der Nutzung: Wie nutzen die Schülerinnen und Schüler die Erklärvideos und wofür? Welche Lernstrategien und -techniken werden eingesetzt? Werden die Erklärvideos für ein den zeitlichen Aufwand minimierendes Lernverhalten (Minimalstrategie) genutzt - also ohne Aufbau von Orientierungswissen, sondern lediglich für ein Verfügungswissen - bzw. ermöglichen Erklärvideos ein Noten maximierendes Lernen bei gleicher Lernzeit (Maximalstrategie)? Oder dienen Erklärvideos einem genuinen Bildungsinteresse der Schülerinnen und Schüler zur interessensgeleiteten Vertiefung des eigenen Verständnisses?

Im Folgenden wird das Forschungsdesign vorgestellt und die Methoden der Datenauswertung beschrieben. 


\section{Forschungsdesign und Datenauswertung}

Im Zeitraum von Mitte 2019 bis Anfang 2020 erfolgten in den beiden Projekten zwei Befragungen, in deren Rahmen mittels standardisierter Fragebögen im Computer Assisted Personal Interview (CAPI) - Verfahren 1401 Schülerinnen und Schüler der Klassenstufen 8 bis 13 an Haupt-, Real- und Gesamtschulen, Oberschulen sowie Gymnasien in Bremen und in der Region Aachen befragt wurden. In diesen Klassenstufen ist zu erwarten, dass die Schülerinnen und Schüler weitgehend selbstständig lernen und eigene Entscheidungen bzgl. ihrer Medienrepertoires treffen können, d. h., dass sowohl die inhaltliche Unterstützung durch die Eltern geringer als auch ihre Medienautonomie grösser als bei jüngeren Schülerinnen und Schülern ist (Feierabend, Rathgeb, und Reutter 2020, 10). Die Befragung erfolgte als Klumpenstichprobe in einzelnen Klassen der beteiligten Schulen als Vollerhebung. Das Sample umfasst keine Schulen in ländlichen Settings, sondern nur in Grossstädten mittlerer Grösse und deren Umland. Die folgende Tabelle 2 fasst die soziodemographischen Daten der befragten Schülerinnen und Schüler zusammen. Die Schulformen verteilten sich auf Gymnasium (37,7\%), Oberschule mit Oberstufe (28,6\%), Oberschule ohne Oberstufe $(7,4 \%)$, Gesamtschule $(13,7 \%)$, Realschule $(11,1 \%)$ und fehlende Zuordnung $(1,6 \%)$.

\begin{tabular}{lccc} 
& \multicolumn{3}{c}{ Gender } \\
\cline { 2 - 4 } & w & m & d \\
\hline $\mathrm{N}$ & 741 & 651 & 9 \\
\hline$(\%)$ & $(52.9)$ & $(46.5)$ & $(0.6)$
\end{tabular}

\begin{tabular}{cccccc}
\multicolumn{7}{c}{ Klassenstufe } \\
\hline 8 & 9 & 10 & 11 & 12 & 13 \\
\hline 36 & 643 & 174 & 382 & 131 & 13 \\
\hline$(2.6)$ & $(45.9)$ & $(12.4)$ & $(27.3)$ & $(9.4)$ & $(0.9)$
\end{tabular}

\begin{tabular}{cc}
\multicolumn{2}{c}{ Stadt/Region } \\
\hline Bremen & Aachen \\
\hline 719 & 682 \\
\hline$(51.3)$ & $(48.7)$
\end{tabular}

Tab. 2.: Soziodemographische Daten der Schülerinnen und Schüler. Anmerkung: $N_{\text {total }}=1401$, bei Klassenstufe $n=22$ fehlende Werte, $w=$ weiblich, $m=$ männlich, $d=$ divers.

Die Befragungsdaten werden zunächst deskriptiv uni- und bivariat in Bezug auf die Fragestellungen ausgewertet (Umfang der Nutzung von Erklärvideos für die Schule, nach Fächern, nach Schulformen, nach Jahrgangsstufen). Es werden weiterhin bivariate Analysen bzgl. der korrelativen Zusammenhänge zwischen Erklärvideonutzung und selbstberichteten Schulnoten durchgeführt und ausgewertet. Basierend auf der Nutzung von Lernstrategien und Motiven zur Nutzung von Erklärvideos werden jeweils Cluster gebildet (Clusteranalyse) sowie in Bezug auf ihre Erklärvideonutzung miteinander verglichen (Varianzanalyse). Wegen der sehr unterschiedlichen Soziallagen der Schulen wurde in diesem Beitrag auf eine nach Schulformen differenzierte Analyse der Daten verzichtet. 


\section{Ergebnisse}

\subsection{Nutzung von Erklärvideos nach Fächern}

Zunächst soll geklärt werden, für welche Schulfächer Erklärvideos besonders häufig rezipiert werden. Die meisten Erklärvideos werden im Kontext des Faches Mathematik geschaut: Für dieses Fach gaben nur 19 \% der Befragten an, «nie» Videos zu schauen, während $48 \%$ aussagten, «mehrfach im Monat» oder häufiger Erklärvideos in Mathematik zu nutzen. Erklärvideos für die Sprachfächer Englisch und Deutsch werden eher weniger rezipiert: Jeweils ca. 60 \% der Schülerinnen und Schüler gaben an, in diesen Fächern «nie» ein Video zu schauen (entsprechend $58 \%$ und $62 \%$ ). Nur $16 \%$ bzw. $11 \%$ der befragten Schülerinnen und Schüler gaben an, für Englisch und Deutsch «mehrmals im Monat» oder häufiger Erklärvideos anzuschauen. Recht häufig werden auch Erklärvideos für die Fächer Biologie und Geschichte genutzt. Selbst für die in der Sekundarstufe nicht durchgehend unterrichteten Fächer Chemie und Physik gaben die Schülerinnen und Schüler an, häufiger Erklärvideos anzuschauen als für die Hauptfächer Deutsch und Englisch. Tabelle 3 gibt an, wie häufig Erklärvideos in den jeweiligen Fächern geschaut werden, sortiert nach absteigender Nennung.

\begin{tabular}{|l|c|c|c|c|c|}
\hline Fach & $M$ & $S D$ & $N$ & $\begin{array}{c}\text { Kendall's } \\
\tau-c\end{array}$ & $p$ \\
\hline Mathematik & 2.64 & 1.23 & 1370 & .09 & .000 \\
\hline Biologie & 2.22 & 1.19 & 1368 & -.03 & .113 \\
\hline Geschichte & 2.09 & 1.23 & 1357 & -.01 & .499 \\
\hline Chemie & 1.83 & 1.09 & 1341 & -.05 & .015 \\
\hline Physik & 1.82 & 1.10 & 1316 & -.05 & .013 \\
\hline Englisch & 1.69 & 1.02 & 1350 & .09 & .000 \\
\hline Erdkunde & 1.61 & 1.01 & 1291 & -.01 & .759 \\
\hline Sonstiges & 1.60 & 1.06 & 1073 & .03 & .142 \\
\hline Sport & 1.60 & 1.12 & 1331 & .05 & .009 \\
\hline Deutsch & 1.56 & 0.88 & 1341 & .06 & .002 \\
\hline Musik & 1.41 & 0.96 & 1270 & .04 & .012 \\
\hline Kunst & 1.32 & 0.81 & 1293 & .03 & .053 \\
\hline Informatik & 1.29 & 0.78 & 1239 & .02 & .100 \\
\hline
\end{tabular}

Tab. 3.: Mittlere Häufigkeit des Schauens von Erklärvideos nach Schulfach und Zusammenhang mit Noten. Anmerkung. Skala $1=$ «nie», $2=$ «weniger als einmal pro Monat», $3=$ «mehrfach im Monat», 4 = «einmal wöchentlich», $5=$ «mehrmals wöchentlich/mehrmals täglich», Kendall's t-c zum Zusammenhang mit Schulnoten; fett $=p<.05$. 
Von besonderem Interesse ist, ob diese Nutzungsintensität mit der formalen Bedeutung der Fächer (gemessen am Stundenumfang oder der Versetzungsrelevanz) einhergeht. Bei einem ressourcenoptimierenden Einsatz würde man davon ausgehen, dass Schülerinnen und Schüler die meiste Lernzeit auf versetzungsrelevante Fächer legen. In den drei Hauptfächern Mathematik, Deutsch und Englisch wird allerdings lediglich Mathematik intensiv rezipiert. Insbesondere Videos zu Geschichte und den Naturwissenschaften Biologie sowie Physik und Chemie werden häufiger genutzt als für die Hauptfächer Deutsch und Englisch. Es besteht also kein direkter Zusammenhang zwischen der formalen Bedeutung der Fächer in der Schule (gemessen am Stundenumfang oder der Versetzungsrelevanz) und der Rezeption und Nutzung von Erklärvideos für die jeweiligen Fächer durch die Schülerinnen und Schüler. Ungeklärt bleibt dabei, ob sich bestimmte Fächer besser für eine Erklärung durch Erklärvideos eignen und somit auch ein grösseres Angebot verfügbar ist, welches dann vermehrt von den Schülerinnen und Schülern genutzt wird, oder ob es für spezifische Fächer einfach mehr Nachfrage gibt, welche zu einem grösseren Angebot führt. Dies könnte für verschiedene Fächer durchaus unterschiedlich zu beantworten sein. So werden von Schülerinnen und Schüler z. B. kaum Informatik-Erklärvideos geschaut, obwohl es eine grosse Anzahl von Informatik-Erklärvideos gibt. Dies hängt in der vorgestellten Studie mit hoher Sicherheit mit dem kaum angebotenen Informatikunterricht an den erhobenen Schulen zusammen.

\subsection{Bedeutung von Erklärvideos in nachhilfeintensiven Fächern}

Dies eröffnet die Frage, ob Erklärvideos besonders häufig für nachhilfeintensive Fächer genutzt werden. Erklärvideos könnten womöglich unabhängig von den finanziellen Ressourcen der Eltern einen Zugang zu unterstützenden Lernressourcen vermitteln und somit Nachteile beim Bildungskapital - insbesondere beim ökonomischen, infrastrukturellen und didaktischen Bildungskapital - ausgleichen (vgl. Kapitel 2 und Tab. 1).

In einem der grössten deutschen Nachhilfeinstitut «Studienkreis» nehmen nach Angaben des Instituts $63 \%$ der Schülerinnen und Schüler Nachhilfe in Mathematik, $30 \%$ in Deutsch und $25 \%$ in Englisch. In allen weiteren Fächern wird nur in geringem Masse (<5\%) Nachhilfe nachgefragt (Studienkreis 2019). In einer Elternbefragung der Bertelsmannstiftung berichten die Eltern von Nachhilfeschülerinnen und -schüler für Mathematik $61 \%$, für Fremdsprachen insgesamt $46 \%$ sowie für Deutsch $31 \%$ (Klemm und Hollenbach-Biele 2016, 17). Diese Daten entsprechen ungefähr einer älteren Studie von Dohmen et al. (2008): 57 \% Mathematik; 33 \% Englisch; 28 \% Deutsch; 9 \% Französisch; 5 \% Naturwissenschaften. 
Für Mathematik deckt sich also die Nachhilfeintensität mit der in unserer Studie berichteten Häufigkeit der Videonutzung. Dies gilt allerdings weniger für die Sprachfächer Englisch und Deutsch. Obwohl beide Fächer nachhilferelevant sind, werden für sie deutlich weniger Erklärvideos rezipiert als für andere Fächer wie z. B. Biologie oder Geschichte, welche keine Bedeutung für die Nachhilfe haben. Ungeklärt bleibt dabei, ob sich Erklärvideos weniger für die Unterstützung von Sprachfächern eignen und deshalb nicht so häufig wie Erklärvideos für Mathematik geschaut werden. So könnte der reine Erklärbedarf bei Sprachfächern geringer sein als in MINT-Fächern - so verzichtet z. B. der erfolgreiche YouTube-Kanal SimpleClub vollständig auf Angebote für Fremdsprachen, da Sprachlernen aus Sicht der Produzierenden von SimpleClub sehr viel Übung und Wiederholungslernen erfordert (Giesecke und Wolf 2020, 40). Ggf. bedarf das Lernen von Sprachfächern im Vergleich zu den anderen Fächern auch vermehrt weitere Bildungs- und Lernkapitalsorten der Haushalte wie didaktisches (insbes. Zugang zu schriftlichen Lernmitteln und Apps) oder soziales Bildungskapital (insbes. Nachhilfe durch Familienangehörige) sowie telisches (insbes. Setzen und Beibehalten von Lernzielen) und attentatives (insbes. Fokussierung von Lernaktivität) Lernkapital. Erklärvideos könnten deshalb für Sprachfächer weniger wirksam für das schulbezogene Lernen sowie eine damit verbundene Leistungsoptimierung sein, und somit auch weniger genutzt werden.

\subsection{Kompensatorische Nutzung von Erklärvideos}

Nachhilfe in Deutschland wird in der betrachteten Altersgruppe überwiegend von leistungsschwächeren Schülerinnen und Schüler - also auf den Notenstufen 4 und 5 - und grösstenteils nicht von sehr guten bis guten Schülerinnen und Schüler in Anspruch genommen (Birkelbach, Dobischat, und Dobischat 2017, 110ff) ${ }^{5}$. Werden Erklärvideos nun überwiegend kompensatorisch genutzt (also eher von schwächeren Schülerinnen und Schülern) oder auch als Werkzeug zur vertiefenden Auseinandersetzung mit dem Schulstoff eingesetzt (eher von stärkeren Schülerinnen und Schülern)?

Zur Klärung dieser Frage wurde zunächst der Effekt der allgemeinen Schulleistung auf die Häufigkeit des Videoschauens in den einzelnen Fächern untersucht (die Werte für den Zusammenhangsmass Kendall's $\tau$-c werden in Tab. 3 berichtet). Hierzu wurden die Angaben zu Noten in Deutsch, Englisch und Mathematik für die Schülerinnen und Schüler jeweils zusammengefasst, um eine Gesamteinschätzung der

5 Laut der Bertelsmann Studie (Klemm und Hollenbach-Biele 2016, 19) haben jedoch ca. ein Viertel der Nachhilfeschülerinnen und -schüler in Mathematik, Deutsch und Fremdsprachen die Note 3, was für ein zusätzliches Motiv der Leistungssteigerung für die Verbesserung der Chancen eines Übergangs in höhere Bildungswege spricht. 
individuellen Schulleistung zu geben ${ }^{6}$. Kein signifikanter Zusammenhang zwischen dem allgemeinen Notenstand und den Häufigkeitsangaben zu Erklärvideos zeigt sich für die Fächer Biologie, Kunst, Informatik, Geographie, Geschichte, und sonstigen Fächern. Das bedeutet, dass Videos für diese Fächer von stärkeren und schwächeren Schülerinnen und Schülern gleichermassen häufig geschaut werden.

Einen signifikanten positiven Zusammenhang ${ }^{7}$ mit dem allgemeinen Notenstand gibt es für die restlichen Fächer: Kompensatorisch werden Videos für Mathematik (Kendall's $\mathrm{t}-\mathrm{c}=.09, \mathrm{p}<.001$ ), Englisch (Kendall's $\mathrm{t}-\mathrm{c}=.09, \mathrm{p}<.001$ ), Deutsch (Kendall's $\mathrm{t}-\mathrm{c}=.06, \mathrm{p}<.01$ ), Sport (Kendall's $\mathrm{t}-\mathrm{c}=.05, \mathrm{p}=.01$ ) und Musik (Kendall's $\mathrm{\tau}-\mathrm{c}=.04$, $p=.01)$ geschaut. Hier lässt sich nun eine Verbindung zu den versetzungsrelevanten Fächern erkennen: Es werden zwar insgesamt eher wenig Videos für Deutsch geschaut, aber die wenigen Schülerinnen und Schüler, die Videos in dem Fach schauen, sind insbesondere schwache Schülerinnen und Schüler. Genau anders herum stellt sich die Nutzung von Erklärvideos für die Fächer Physik und Chemie dar: Diese beiden Fächer werden signifikant häufiger von stärkeren Schülerinnen und Schülern genannt (Kendall's $\mathrm{t}-\mathrm{c}=-.05, \mathrm{p}=.01$ und Kendall's $\mathrm{t}-\mathrm{c}=-.05, \mathrm{p}=.02$ ), was auf einen Einsatz zum vertieften Lernen hindeuten könnte. Es könnte aber auch einen indirekten Effekt der Fächerwahl von stärkeren Schülerinnen und Schülern darstellen, da anzunehmen ist, dass insgesamt schwächere Schülerinnen und Schüler die Fächer Physik und Chemie gar nicht belegen (können) und somit sowieso nur allgemein gute Schülerinnen und Schüler Erklärvideos für Physik und Chemie rezipieren, egal, ob sie gut oder schlecht in den beiden Fächern sind.

Der Zusammenhang von Schulleistung im jeweiligen Fach mit der konkreten fachbezogenen Videonutzung wurde zusätzlich einzeln für die versetzungsrelevanten Hauptfächer (Mathematik, Deutsch, Englisch) analysiert. Hier zeigt sich ebenfalls ein signifikanter kompensatorischer Effekt für alle drei Fächer Mathematik (Kendall's $\mathrm{T}-\mathrm{c}=.19, \mathrm{p}<.001$ ), Englisch (Kendall's $\mathrm{t}-\mathrm{c}=.13, \mathrm{p}<.001$ ) und Deutsch (Kendall's $\tau-c=.07, p=.001$ ) - schlechte Schülerinnen und Schüler berichteten von einer stärkeren Nutzung. Der beobachtete Zusammenhang zwischen Fachleistung und Videonutzung ist dabei für die Fächer Mathematik und Englisch höher als zwischen allgemeinem Notenniveau und Videonutzung, was auf eine fachbezogene Mehrnutzung zur Kompensation schlechterer Noten schliessen lässt. Abbildungen 1 bis 3 zeigen die Verteilung der Häufigkeitsangaben jeweils innerhalb der drei Notengruppen für die einzelnen Fächer.

6 In der Erhebung wurden Selbstangaben zu den Noten in den Fächern Deutsch, Englisch und Mathematik erhoben (Skala: Richtung 1-2; um 3; Richtung 4-5).

7 Da höhere Notenstufen schlechtere Noten bedeutet (Notenskala 1-5), bedeutet ein positiver Zusammenhang, dass eine höhere Nutzung von Erklärvideos mit einer schlechteren Note einhergeht. 
Rezeption von Erklärvideos für das Fach Mathematik

Zustimmung

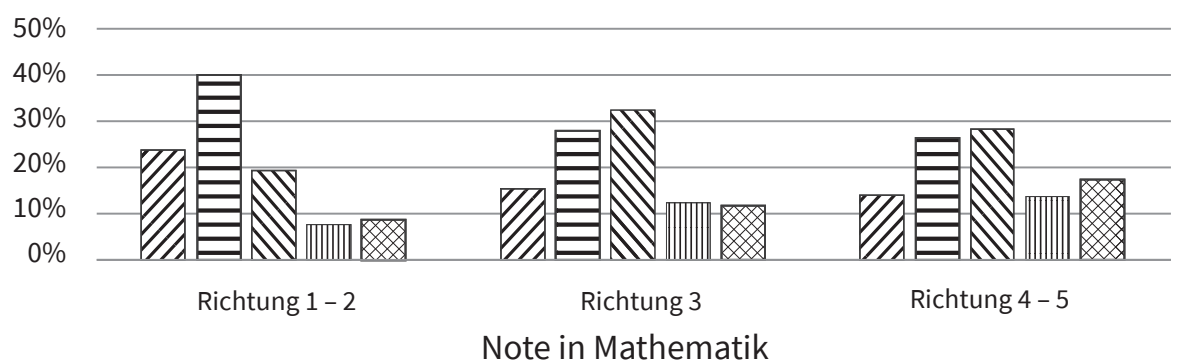

\footnotetext{
$\nabla /$ nie

weniger als einmal pro Monat

NV mehrfach im Monat

|d|||| einmal wöchentlich

mehrmals wöchentlich bis mehrmals täglich
}

Abb. 1.: Häufigkeit des Schauens von Erklärvideos in Mathematik innerhalb einer Notengruppe in Mathematik.

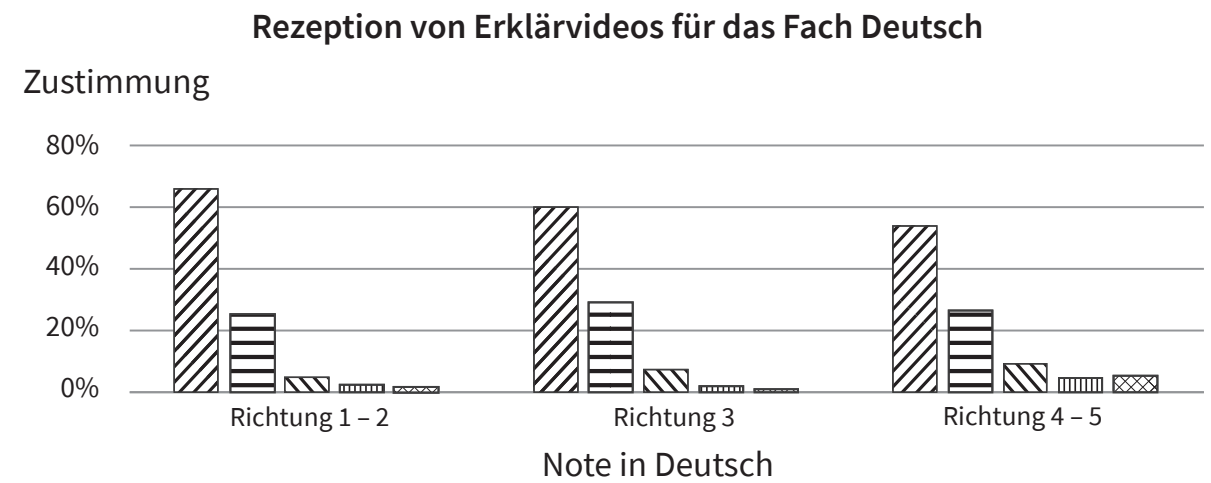

$\mathbb{Z}$ nie

weniger als einmal pro Monat

NIV mehrfach im Monat

||l||| einmal wöchentlich

mehrmals wöchentlich bis mehrmals täglich

Abb. 2.: Häufigkeit des Schauens von Erklärvideos in Deutsch innerhalb einer Notengruppe in Deutsch. 
Rezeption von Erklärvideos für das Fach Englisch

Zustimmung

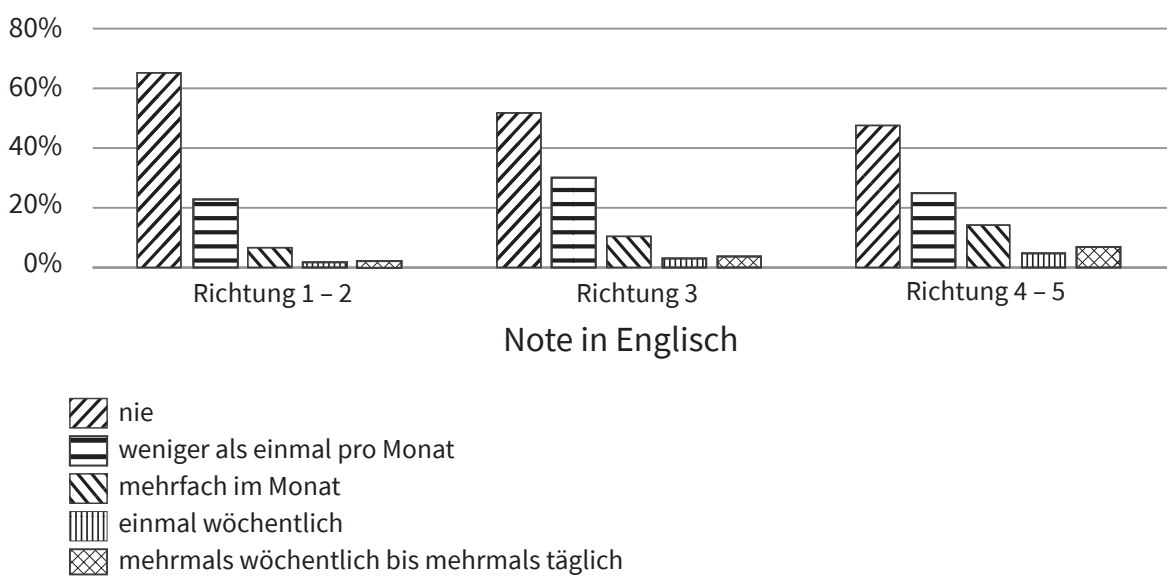

Abb. 3.: Häufigkeit des Schauens von Erklärvideos in Englisch innerhalb einer Notengruppe in Englisch.

In weiteren Studien muss geklärt werden, ob es dabei einen moderierenden Effekt von genutzter Nachhilfe gibt. Da in der Studie nicht erhoben wurde, ob die Schülerinnen und Schüler in den einzelnen Fächern Nachhilfe hatten, ist nicht zu klären, ob ggf. schlechte Schülerinnen und Schüler ohne Nachhilfe mehr, gleich oder weniger Erklärvideos in dem Fach schauen als schlechte Schülerinnen und Schüler mit Nachhilfe.

\subsection{Motivationale Gründe und Nutzungsanlässe zur unterrichtsbezogenen Nutzung von Erklärvideos}

Neben der Häufigkeit der Nutzung ist die Frage nach den Nutzungsanlässen und motivationalen Gründen der Nutzung von Erklärvideos relevant. Werden die Erklärvideos im Sinne des Minimalprinzips überwiegend zur lernzeitminimierenden Absicherung eines angestrebten Notenniveaus genutzt oder sind sie Bestandteil einer Maximalstrategie zur (zusätzlichen) Vertiefung oder Wiederholung des bereits verstandenen Stoffes? Welchen Einfluss haben ggf. andere Personen auf die Rezeption von Erklärvideos durch die Schülerinnen und Schüler?

Die befragten Schülerinnen und Schüler stimmen eher Gründen zu, die sich direkt auf den Inhalt des Schulstoffes beziehen («Um Dinge besser zu verstehen», «Wissensvertiefung» oder «Weil mich das Thema interessiert») sowie den Unterricht («Für bessere Noten», «Lehrkraft erklärt schlecht»). 


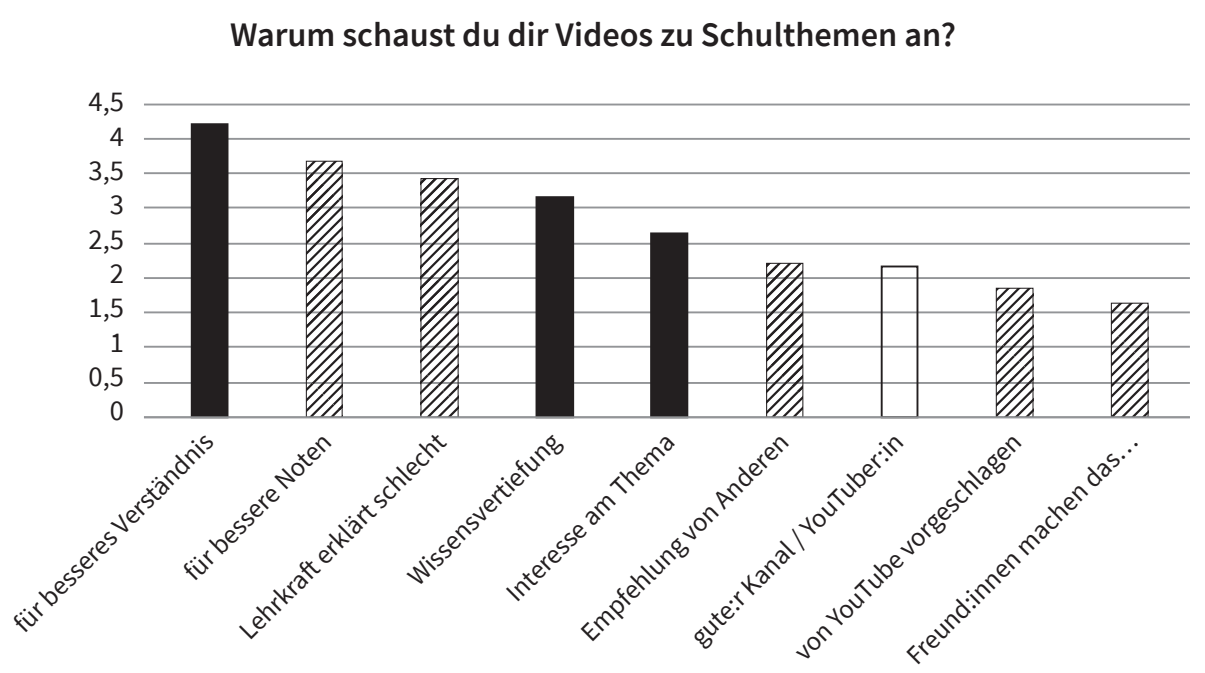

Abb. 4.: Mittlere Zustimmung der selbst- und fremdbestimmten Videoschaugründe (Selbstbestimmte Gründe sind schwarz eingefärbt, fremdbestimmte Gründe schraffiert; Skala von 1 «stimme überhaupt nicht zu» bis 5 «stimme völlig zu»).

Die Gründe, eine bessere Note zu erzielen bzw. wegen der schlechten Erklärung durch die Lehrperson, können als allgemein optimierungsbezogene Gründe interpretiert werden. Die beiden anderen häufig genannten Gründe «besseres Verständnis» und «Wissensvertiefung» sprechen für eine tiefergehende Auseinandersetzung mit dem Schulstoff im Sinne einer Maximalstrategie. Dem optimierungsfremden Grund «Interesse am Thema» wird in geringerem Masse zugestimmt.

Bei einer leistungsdifferenzierten Analyse zeigen sich signifikante Effekte der Schulleistung (siehe Tab. 4): stärkere Schülerinnen und Schüler stimmen eher zu, Videos zu schauen, um Dinge besser zu verstehen (Kendall's $t-c=-.05, p=.02$ ) / weil das Thema interessiert (Kendall's $\mathrm{T}-\mathrm{c}=-.10, p<.000$ ) / um Wissen vertiefen (Kendall's $T-c=-.08, p<.000$ ) als schwächere Schülerinnen und Schüler (siehe Tabelle 4). Alle drei Gründe, bei denen sich signifikante Unterschiede nach Schulleistung zeigen, können im Rahmen der Selbstbestimmungstheorie nach Ryan und Deci (2017) eher selbstbestimmten Strategien der Motivationsregulation zugeordnet werden, die das Bedürfnis nach Kompetenzempfinden und Autonomie unterstützen. Bessere Schülerinnen und Schüler schauen also mehr aus selbstbestimmten Gründen schulbezogene Videos als Schülerinnen und Schüler im unteren Leistungsbereich. Bei den fremdbestimmten Motiven gibt es keine leistungsbezogenen Unterschiede. 


\begin{tabular}{|l|c|c|c|c|c|}
\hline $\begin{array}{l}\text { «Warum schaust du dir Videos zu Schulthe- } \\
\text { men an?» }\end{array}$ & $M$ & $S D$ & $N$ & $\begin{array}{c}\text { Kendall's } \\
\tau-\mathrm{c}\end{array}$ & $p$ \\
\hline Um Dinge besser zu verstehen (SB) & 4.20 & 1.08 & 1382 & -.05 & .018 \\
\hline Weil ich bessere Noten haben möchte (FB) & 3.70 & 1.31 & 1382 & .03 & .206 \\
\hline $\begin{array}{l}\text { Weil der Lehrer/die Lehrerin schlecht erklärt } \\
\text { (FB) }\end{array}$ & 3.42 & 1.26 & 1383 & .03 & .210 \\
\hline Um mein Wissen zu vertiefen (SB) & 3.16 & 1.39 & 1374 & -.08 & .000 \\
\hline Weil mich das Thema interessiert (SB) & 2.64 & 1.35 & 1382 & -.10 & .000 \\
\hline $\begin{array}{l}\text { Weil mir das empfohlen wurde (Lehrerin \& } \\
\text { Lehrer, Eltern, Nachhilfe...) (FB) }\end{array}$ & 2.20 & 1.23 & 1382 & .04 & .080 \\
\hline Weil ich den Kanal/YouTuber*in gut finde (-) & 2.16 & 1.33 & 1374 & -.01 & .507 \\
\hline $\begin{array}{l}\text { Weil mir das von YouTube vorgeschlagen } \\
\text { wurde (FB) }\end{array}$ & 1.84 & 1.08 & 1382 & .02 & .415 \\
\hline $\begin{array}{l}\text { Weil meine Freunde und Freundinnen das } \\
\text { auch machen (FB) }\end{array}$ & 1.63 & .93 & 1374 & .01 & .615 \\
\hline
\end{tabular}

Tab. 4.: Mittlere Zustimmung und zu selbst- versus fremdbestimmten Gründen, Videos zu Schulthemen zu schauen und Zusammenhang zu Schulnoten. Anmerkung: Fünfstufige Antwortskala von 1 = «stimme überhaupt nicht zu» bis 5 = «stimme völlig zu», SB = selbstbestimmt, FB = fremdbestimmt; Kendall's $\mathrm{T}$-c zum Zusammenhang von Schulnoten und Gründen.

Insgesamt weniger relevant für die Auswahl und das Schauen von Erklärvideos sind Empfehlungen von Lehrpersonen. Kaum eine Rolle spielen Empfehlungen durch die Plattform YouTube selbst ${ }^{8}$ oder die Handlungspraxen der Peers. Die Ergebnisse deuten auf ein fokussiertes Suchen durch die Schülerinnen und Schüler hin, welche sich für jeweils spezifische unterrichtsrelevante Themen Erklärvideos anschauen.

Zusammenfassend lässt sich also sagen, dass die motivationalen Gründe zur Nutzung von Erklärvideos zu schulbezogenen Themen überwiegend leistungsoptimierender Natur sind. Eine vertiefende Nutzung im Sinne einer Maximalstrategie bzw. aus Interesse erfolgt aber mehr von den besseren Schülerinnen und Schülern.

Gefragt nach konkreten unterrichtsbezogenen Nutzungsanlässen von Erklärvideos geben $80 \%$ der Befragten an, Erklärvideos mindestens «ab und zu» zur Klausur- und Prüfungsvorbereitung zu nutzen, $65 \%$ wenn sie etwas nicht ausreichend verstanden haben und $56 \%$ als Hilfe für Hausaufgaben (siehe Tab. 5 und Abb. 5). Nur $32 \%$ nutzen Erklärvideos «wenn ein neues Thema im Unterricht kommt».

Zur Klärung von Verständnislücken sowie bei der Hilfe bei Hausaufgaben unterscheiden sich die Schülerinnen und Schüler bezogen auf ihre Schulleistung nicht. Erklärvideos sind somit fest im schulbezogenen Medienrepertoire von Schülerinnen und Schüler aller Leistungsstufen etabliert. Nur für die Aussage «Wenn ein neues

8 Dabei wird unter «Empfehlungen durch die Plattform YouTube» eine direkte Listung von Erklärvideos auf der Startseite von YouTube verstanden, also ohne konkrete Suchanfrage (siehe auch Kapitel 4.5 zu Techniken und Lernstrategien). 
Thema im Unterricht kommt» kann ein signifikanter positiver Zusammenhang (eher schwächere Schülerinnen und Schüler stimmen zu) berichtet werden (siehe Tab. 5 und Abb. 5).

\begin{tabular}{|l|c|c|c|c|c|}
\hline $\begin{array}{l}\text { «Wann schaust du dir Erklärvideos/Tutori- } \\
\text { als zu Schulthemen an?» }\end{array}$ & $M$ & $S D$ & $N$ & $\begin{array}{c}\text { Kendall's } \\
\text { T-c }\end{array}$ & $p$ \\
\hline Wenn bald ein Test/eine Klausur kommt. & 3.67 & 1.32 & 1395 & .04 & .055 \\
\hline $\begin{array}{l}\text { Wenn ich was nicht verstanden habe (Ver- } \\
\text { ständnislücke). }\end{array}$ & 3.05 & 1.33 & 1395 & .01 & .792 \\
\hline $\begin{array}{l}\text { Wenn ich Hilfe bei den Hausaufgaben brau- } \\
\text { che. }\end{array}$ & 2.82 & 1.35 & 1395 & .01 & .797 \\
\hline $\begin{array}{l}\text { Wenn ein neues Thema im Unterricht } \\
\text { kommt. }\end{array}$ & 2.11 & 1.20 & 1395 & .05 & .032 \\
\hline
\end{tabular}

Tab. 5.: Unterrichtsbezogene Nutzungsanlässe von Erklärvideos. Anmerkung. Fünfstufige Antwortskala von $1=$ «nie» bis $5=$ «immer»; Kendall's $t-c$ zum Zusammenhang von Schulnoten und Gründen.

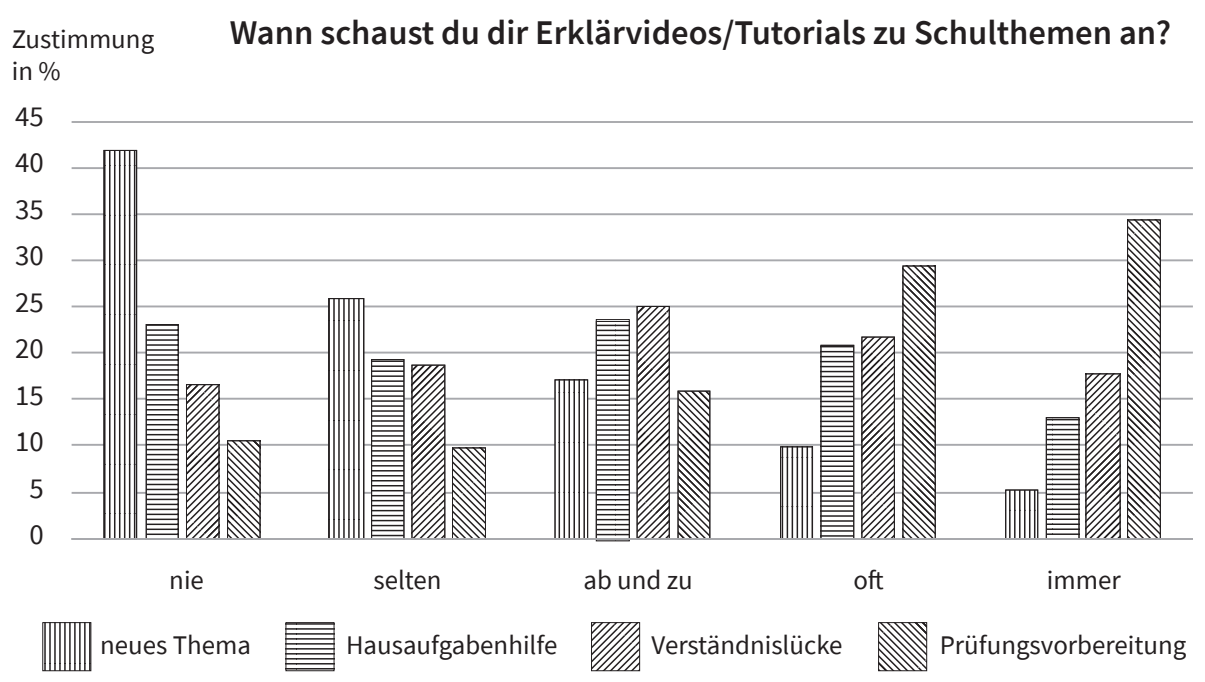

Abb. 5.: Zustimmung zu Nutzungsanlässen für Erklärvideos in Prozent (\%).

\subsection{Techniken und Lernstrategien bei der Videonutzung}

Ein Vorteil bei der Rezeption von Erklärvideos stellt die Möglichkeit der Videosteuerung dar (Wolf 2015a, Wolf und Kulgemeyer 2021). Dies ermöglicht - ganz im Gegensatz zum Lehrvortrag im Klassenzimmer - die Erklärung individuell zu pausieren, zurück zu spulen oder beliebig häufig zu wiederholen. Knapp $80 \%$ der Befragten geben an, mindestens «ab und zu» ein Erklärvideo zu pausieren oder vor- und zurück zu spulen. Hingegen wurden Untertitel und Geschwindigkeitsänderungen eher weniger genutzt: hier gaben jeweils ca. 90 \% der Schülerinnen und Schüler an, diese Technik 
nie oder selten einzusetzen. Auch Zusammenhänge zu Schulnoten waren nicht signifikant - schwache und starke Schülerinnen und Schüler gaben ähnliche Nutzungstechniken an (siehe Abb. 6 und Tab. 6).

\begin{tabular}{|l|c|c|c|c|}
\hline $\begin{array}{l}\text { «Während ich ein Video zu Schulthemen } \\
\text { schaue... }\end{array}$ & $M$ & $S D$ & $\begin{array}{c}\text { Kendall's } \\
\text { T-c }\end{array}$ & $p$ \\
\hline ...spule ich vor oder zurück» & 3.42 & 1.21 & .01 & .673 \\
\hline ...drücke ich auf Pause» & 3.31 & 1.18 & -.01 & .504 \\
\hline ...schalte ich die Untertitel ein» & 1.47 & .91 & .01 & .561 \\
\hline ...ändere ich die Geschwindigkeit» & 1.32 & .76 & -.00 & .870 \\
\hline
\end{tabular}

Tab. 6.: Umgang mit Erklärvideos und Zusammenhang mit den Noten. Anmerkung. $N=1392$; Fünfstufige Antwortskala von 1 = «nie» bis 5 = «immer»; Kendall's t-c zum Zusammenhang von Schulnoten und Gründen.

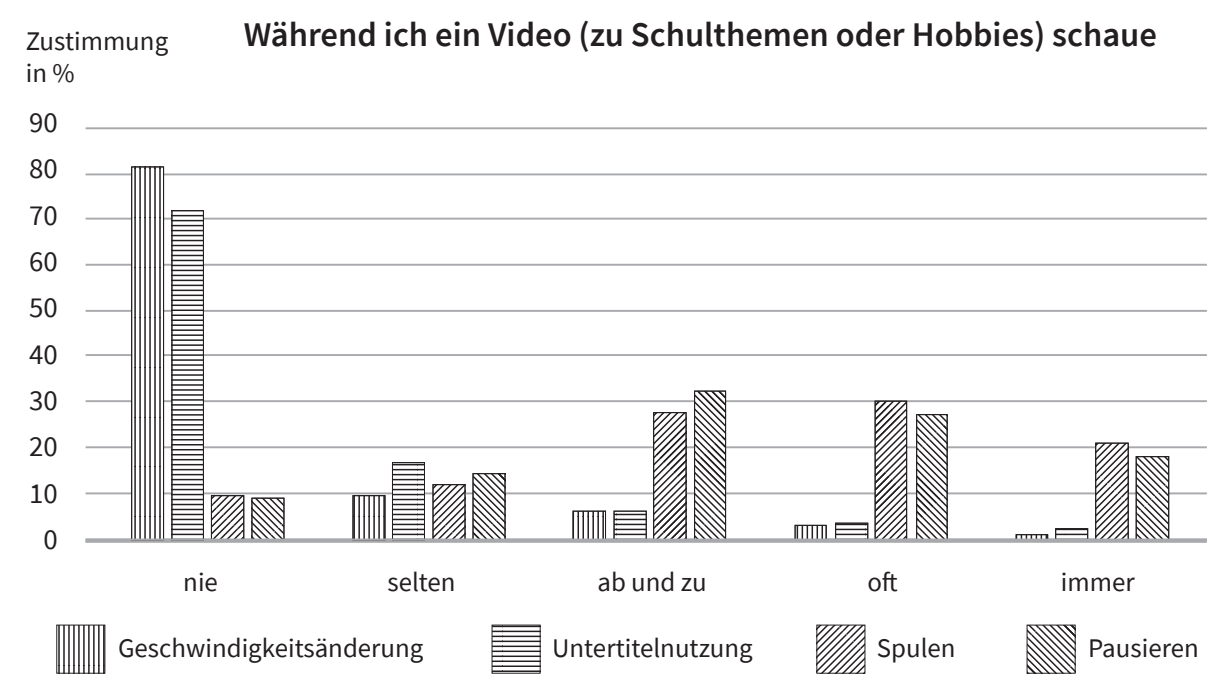

Abb. 6.: Zustimmung zu Nutzungstechniken von Erklärvideos in Prozent.

Abschliessend soll analysiert werden, ob die Erklärvideos eher für ein zeitoptimierendes Lernverhalten genutzt - also ohne Aufbau von Orientierungswissen und ohne eine verstehensorientierte Auseinandersetzung mit den Lerninhalten - und damit lediglich dem Aufbau eines Verfügungswissens unter Ausklammerung des Bildungseffektes dienen. Zur Beantwortung dieser Fragestellung wurde auf Grundlage des Fragebogens «Lernstrategien im Studium» (LIST; Schiefele und Wild 1994) eine Kurzversion «Lernstrategien bei der Nutzung von Erklärvideos und Tutorials» (Wolf et al. 2019) entwickelt (siehe Tabelle 7).

Tabelle 7 zeigt die mittlere Nutzungshäufigkeit von Lernstrategien auf Faktor- und Itemebene. Die Faktorstruktur wurde mittels konfirmatorischer Faktoranalyse bestä$\operatorname{tigt}\left(\mathrm{X}^{2}(62)=526.34, p=.000, \mathrm{CFI}=.92, \mathrm{RMSEA}_{\mathrm{CI} 95}=[.07 ; .08], p_{\mathrm{RMSEA}<=.05}=.000\right)$. So gaben die befragten Schülerinnen und Schüler an, am häufigsten kognitive Lernstrategien 
einzusetzen $(M=2.94, S D=.97)$, gefolgt von Strategien zum ressourcenorientierten Lernen $(M=2.52, S D=.83)$. Am wenigsten werden metakognitive Strategien eingesetzt $(M=2.25, S D=.96)$. Auf Skalenebene unterscheidet sich der Lernstrategieeinsatz der Schülerinnen und Schüler in Bezug auf ihr Notenniveau nicht.

\begin{tabular}{|c|c|c|c|c|c|}
\hline & $\begin{array}{l}\text { Wenn du YouTube nutzt, um etwas zu lernen, wie } \\
\text { machst du das? }\end{array}$ & M & $S D$ & $\begin{array}{c}\text { Kendall's } \\
\tau-c\end{array}$ & $p$ \\
\hline \multicolumn{6}{|c|}{ Subskalenebene } \\
\hline $\mathrm{KL}$ & Kognitive Lernstrategien (KL) & 2.94 & .97 & -.02 & .288 \\
\hline MKL & Metakognitive Lernstrategien (MKL) & 2.25 & .96 & .02 & .332 \\
\hline ROL & Ressourcen Orientiertes Lernen (ROL) & 2.52 & .83 & .02 & .417 \\
\hline \multicolumn{6}{|c|}{ Itemebene } \\
\hline ROL & $\begin{array}{l}\text { Ich suche nach weiteren Videos, wenn mir etwas noch } \\
\text { nicht klar ist. (Externe Ressourcen) }\end{array}$ & 3.46 & 1.33 & -.02 & .342 \\
\hline $\mathrm{KL}$ & Ich schaue mir das Video mehrmals an. (Wiederholen) & 3.35 & 1.25 & .03 & .224 \\
\hline $\mathrm{KL}$ & $\begin{array}{l}\text { Ich denke über den Zusammenhang zwischen dem Vi- } \\
\text { deo und meinem Schulstoff nach. (Zusammenhänge) }\end{array}$ & 3.32 & 1.31 & -.10 & .000 \\
\hline $\mathrm{KL}$ & Ich mache mir Notizen zum Video. (Organisation) & 2.85 & 1.40 & .02 & .328 \\
\hline KL & Ich mache mir Screenshots. (Organisation) & 2.61 & 1.39 & .10 & .000 \\
\hline MKL & $\begin{array}{l}\text { Die Sachen, die ich nicht verstanden habe, notiere ich } \\
\text { mir, um sie mir danach zu erarbeiten. }\end{array}$ & 2.55 & 1.33 & .02 & .377 \\
\hline $\mathrm{KL}$ & $\begin{array}{l}\text { Ich frage mich, ob der Inhalt des Videos wirklich rich- } \\
\text { tig ist. (Kritisches Prüfen) }\end{array}$ & 2.41 & 1.23 & -.03 & .168 \\
\hline MKL & $\begin{array}{l}\text { Ich stelle mir nach dem Videoschauen Fragen, um } \\
\text { sicher zu gehen, dass ich den Inhalt verstanden habe. }\end{array}$ & 2.41 & 1.31 & .02 & .298 \\
\hline ROL & $\begin{array}{l}\text { Ich beginne frühzeitig mit dem Lernen und suche mir } \\
\text { Erklärvideos, damit ich nicht in Zeitnot gerate. (Inter- } \\
\text { ne Ressourcen) }\end{array}$ & 2.23 & 1.18 & -.03 & .252 \\
\hline$-\mathrm{ROL}$ & $\begin{array}{l}\text { Beim Erklärvideos schauen merke ich, dass ich durch } \\
\text { andere Videos abgelenkt werde. (Interne Ressourcen) }\end{array}$ & 2.20 & 1.20 & .09 & .000 \\
\hline ROL & $\begin{array}{l}\text { Wenn ich etwas in den Videos nicht verstehe, frage } \\
\text { ich meine Lehrerin oder meinen Lehrer, meine Eltern } \\
\text { oder Nachhilfelehrerinnen und Nachhilfelehrer. (Ex- } \\
\text { terne Ressourcen) }\end{array}$ & 2.19 & 1.25 & .00 & .877 \\
\hline MKL & $\begin{array}{l}\text { Ich erkläre den dargestellten Inhalt einem Freund/ } \\
\text { einer Freundin um zu sehen, ob ich alles verstanden } \\
\text { habe. }\end{array}$ & 2.06 & 1.20 & -.01 & .834 \\
\hline MKL & $\begin{array}{l}\text { Ich überlege mir vorher eine Reihenfolge, in der ich } \\
\text { Erklärvideos schauen möchte. }\end{array}$ & 2.00 & 1.21 & .03 & .187 \\
\hline
\end{tabular}

Tab. 7.: Lernstrategien bei der Nutzung von Erklärvideos nach Häufigkeit und Zusammenhang zu den Schulnoten (Einzelitems geordnet nach Nutzungshäufigkeit). Anmerkung: $N=1398$ bzw. 1397, fünfstufige Antwortskala von $1=$ «nie» bis $5=$ «immer»; fett $=p<.05$. 
Die am häufigsten genutzte Strategie «Ich suche nach weiteren Videos, wenn mir etwas noch nicht klar ist» ( $M=3.46, S D=1.33)$ belegt einen zentralen Vorteil von YouTube als audio-visuelle Enzyklopädie (Wolf 2015a), welche durch ein grosses Angebot alternativer Erklärangebote zusätzliche Wege zum Verständnis ermöglicht - ganz im Gegensatz zum Unterricht, in dem man auf eine Lehrperson angewiesen ist. Auch das wiederholte Anschauen von Videos $(M=3.35, S D=1.25)$ stell einen immanenten Vorteil einer Videoaufzeichnung gegenüber einem Lehrvortrag im Klassenkontext dar (Wolf und Kulgemeyer 2021). Gute Schülerinnen und Schüler denken eher über den Zusammenhang zwischen dem betrachteten Erklärvideo und dem jeweiligen Schulstoff nach $(M=3.32, S D=1.31)$.

Die Schülerinnen und Schüler nutzen weitere kognitive Lernstrategien wie Notizen oder Screenshots machen, wobei das Screenshots-Machen mehr von schwächeren Schülerinnen und Schülern berichtet wird. Metakognitive Lernstrategien werden nur auf einem mittleren bis niedrigen Niveau berichtet. Insgesamt zeigen die Ergebnisse einen Bedarf, Schülerinnen und Schüler anzuleiten, mehr metakognitive und kognitive Lernstrategien bei der Rezeption von schulbezogenen Erklärvideos zu nutzen.

Ein häufig von Lehrpersonen befürchtetes Ablenkungspotential der Nutzung von YouTube wird durch den Selbstbericht der Schülerinnen und Schüler etwas entkräftet: eine Ablenkung durch andere Videos erfolgt nur mässig $(M=2.20, S D=1.20)$, allerdings häufiger bei schwächeren Schülerinnen und Schülern.

\subsection{Beschränkungen der vorliegenden Studie}

Bezüglich der Beschränkungen der vorliegenden Studie ist zunächst die auf Bremen und die Region Aachen beschränkte Klumpenstichprobe (Vollerhebungen von jeweils mehreren Klassen nach Verfügbarkeit in zufällig ausgewählten Schulen) zu nennen, deren Umfang auf Grund der Projektmittel begrenzt war. Repräsentative Aussagen zu ländlichen Regionen oder zu allen Schülerinnen und Schülern in Deutschland können nicht aus den Daten abgeleitet werden.

Bezüglich der statistischen Analyse konnten die Mittelwerte für die weitere uniund bivariate Analyse lediglich auf Basis der ordinalen Skalen der Nutzungshäufigkeit von Erklärvideos gebildet werden. Für die Einschätzung des Notenniveaus wurden Selbstberichtsdaten der Schülerinnen und Schüler erhoben, welche nicht durch die Lehrpersonen oder Zeugnisse validiert werden konnten. Eine nach den einzelnen Schulformen differenzierte Analyse war auf Basis des begrenzten Samples in der Studie nicht angelegt, könnte aber in weiteren Auswertungen explorativ als Basis für weitere Studien durchgeführt werden. Problematisch erscheinen dabei jedoch die Vielfältigkeit der in der Studie teilgenommenen sechs Schulformen an den zwei 
Standorten (Gymnasium G8, Gymasium G9, Realschule, Gesamtschule, Oberschule mit Oberstufe und Oberschule ohne Oberstufe) sowie der hohe Zusammenhang zwischen sozio-ökonomischer Lage und Schulform in Teilen des Samples.

\section{Selbsthilfe oder Entschulungsstrategie?}

Die vorliegenden Daten zeigen zunächst einmal eindrücklich, dass die Rezeption von Erklärvideos bereits vor den pandemiebedingten Schulschliessungen (und dem daraus folgenden Notfallunterricht) fest im alltäglichen schulbezogenen Handlungsund Medienrepertoire der Schülerinnen und Schüler etabliert war. Aus der Perspektive vieler Lernenden stellt ihre Nutzung damit eine kaum hinterfragte Normalität dar. Ganz im Sinne der vom Gesamtsystem zumindest implizit geforderten Optimierung des Ressourceneinsatzes zur Erzielung eines möglichst guten Ergebnisses (im Sinne einer (guten) Note) kann die Nutzung von Erklärvideos und Tutorials damit zunächst einmal als eine Optimierungsstrategie verstanden werden. Die Strukturen der digitalen und vernetzten Medien ermöglichen eine stets verfügbare Ergänzung des schulischen Erklärangebotes durch Erklärvideos und Tutorials insbesondere auf YouTube, wenn im institutionellen Kontext des Bildungssystems die «professionellen> Erklärenden versagen oder zumindest aus der jeweils individuellen Perspektive und Lernlage (z. B. Vorwissen, Motivation, weitere persönliche oder soziokulturelle Voraussetzungen im Sinne von Lern- und Bildungskapital) der Schülerinnen und Schüler nicht die notwendige Performanz oder Unterstützung liefern.

Auf den ersten Blick unterscheidet sich dies zunächst wenig von der Situation in der jüngeren Vergangenheit. Die Nutzung zusätzlicher Ressourcen zur Unterstützung des schulischen Lernens ist fest etabliert, allerdings in fester Kopplung an das ökonomische Bildungskapitel der Familien. Neben dem Nachhilfemarkt mit einem geschätzten Marktvolumen in Deutschland von ca. 879 Millionen $€$ (Bertelsmann Stiftung 2016) werden von einschlägigen Verlagen und Anbietern durch Übungshefte und andere Lernhilfen im Segment Schule und Lernen laut Angaben von Media Control weitere 446 Million $€$ (STARK Verlag o.J.) über den stationären Buchhandel umgesetzt. Dies entspricht durchschnittlich ca. $40 €$ pro Schülerin bzw. Schüler pro Jahr für Übungswerke - unabhängig von einer potentiellen Lehrmittelfreiheit im jeweiligen Bundesland.

Die von uns berichteten Nutzungsdaten und Selbstaussagen der Schülerinnen und Schüler könnten deshalb als Hinweis darauf gelesen werden, dass hier lange etablierte Strukturen ins Wanken geraten und eine Abstimmung mit den Füssen (bzw. der Aufmerksamkeit) zu beobachten ist. Schülerinnen und Schüler können nun - weitgehend unabhängig vom ökonomischen Kapital ihrer Eltern - auf der Internet-Plattform YouTube hoch individualisiert und bedarfsorientiert Erklärvideos und Tutorials für die Schule nutzen (soweit sie über die notwendigen Kompetenzen 
verfügen). Der niedrigschwellige Zugang zu dieser neuen Form von Erklärressource hat dabei das Potenzial, das klassische Generationengefüge auszuhebeln: die <ältere Generation` im Sinne Schleiermachers verliert so womöglich ihre Schlüssel- und Filterfunktion für Bildung - da das akkumulierte (Schul-)Wissen der Eltern und Lehrpersonen aktualisiert und gut präsentiert auf YouTube angeboten wird. Das formale Bildungssystem hat sein Erklärmonopol mit hoher Wahrscheinlichkeit streckenweise bereits eingebüsst.

Verliert das Schulsystem bzw. die Kultusministerien dadurch aber auch ihre curriculare Gestaltungsmacht? Im Kontext von Schule ist dies (noch) zu bezweifeln. Zwar werden die erfolgreichsten Erklärvideo-Kanäle (Anzahl der Subscriber Anfang Dezember 2021 in Tausendern als K angegeben) wie "SimpleClub Mathe» (816K), «SimpleClub Bio» (588K), «Mathe by Daniel Jung» (831K) oder «MrWissen2go Geschichte» (840K) nahezu ausschliesslich von einer jungen Generation von YouTubern ohne eine formale pädagogische Ausbildung betrieben und nutzen im Kontrast zum Schulunterricht einen an der aktuellen Ästhetik von YouTube orientierten Gestaltungs- und Erklärstil (Wolf 2015a). Und auch die Kanäle von <echten> Lehrpersonen wie «Lehrerschmidt» (1240K) oder die «LehrerBros - Mathe leichtgemacht» (15K) folgen ebenfalls den gängigen YouTube-Konventionen, auch wenn sie tendenziell etwas <ernsthafter gestaltet sind.

Inhaltlich aber orientieren sich alle auf das schulische Feld zielenden Erklärvideokanäle stark an den curricularen Vorgaben der Schule. Gerade die enge Passung zum Schulstoff kombiniert mit einer die Lernzeit (scheinbar) minimierenden Gestaltung der Erklärvideos trägt neben einem lockeren Vermittlungsstil, sympathischen Persönlichkeiten und anschaulicher Visualisierung wesentlich zur Attraktivität dieser Kanäle für die Masse der Schülerinnen und Schüler bei.

Mit Blick auf die oben zitierte Bildungs-Utopie von Illich (1971) zeigen die Daten das aktuelle Bildungsgeschehen als ein Zwitterwesen: Anders als in den kritisch-utopisch-revolutionären Texten der 1970er erhofft, liegt die Hegemonie über die Bestimmung von «relevanten> Inhalten wie auch die Ausgabe von lebenslaufentscheidenden Zertifikaten noch im Herrschaftsbereich des formalen Bildungssystems. Das alltägliche Lernhandeln ist dagegen geprägt von einer intensiven Nutzung von Bildungsangeboten verschiedenster Anbieter, die sich als (mehr oder weniger kostenpflichtiger) Markt neben der Schule etablieren. Je mehr dann im formalen Bildungssystem Misserfolgserlebnisse die Wahrnehmung dominieren und zugleich im non-formalen Feld Erfolgserlebnisse generiert werden, desto schwieriger wird es werden, die alte Hegemonie aufrecht zu erhalten. Die von Illich vorgeschlagenen «lizensierten`Anbietenden als mögliche Intermediäre sind bisher nicht in Sicht. Für die Zukunft bleibt damit fraglich, inwieweit das staatliche Schulsystem neben der Enkulturation unangefochten die ihm nach Fend (2009) zugeschriebenen tradierten Funktionen Allokation, Qualifikation und Integration weiter durchsetzen kann. Die technisch-medialen 
Strukturen der «digitalen» Gegenwart verschieben die Machtpositionen hin zu anderen, insbesondere wirtschaftlichen Akteuren. Qualifikation und damit implizit auch Allokation werden zunehmend losgelöst vom staatlichen Bildungssystem organisiert. So bietet z. B. Googles Mutterkonzern Alphabet Kurse mit eigener Zertifizierung an, um zukünftig diese Zertifikate auch als universitäre Abschlüsse adäquat anzuerkennen (Seele 2020).

Im Kontext schulischer Erklärvideos und Tutorials ist also bisher nicht von einer graswurzelhaften Selbstermächtigung im Sinne einer Bricolage (Lévi-Strauss 1973) der eigenen Bildungsbiographie auszugehen. Vielmehr entsteht der Eindruck einer selbstverantworteten Optimierung im Sinne neoliberaler, hochleistungsorientierter Selbstformierung. Profitieren dürften am Ende dann wieder diejenigen Lernenden, die bereits mit vielfältigen Ressourcen ins Rennen gehen. Ein staatliches Bildungssystem wird deshalb gerade auch in einer digitalen Kultur weiterhin gefordert sein, auch die weniger gut mit Ressourcen (Bildungs- und Lernkapital, Medienkompetenz etc.) ausgestatteten Schülerinnen und Schüler zu unterstützen. Ansonsten wird es (entgegen der vielfach formulierten Intentionen) eher dazu beitragen, soziale Ungleichheit trotz eines durch die Corona-Pandemie induzierten Digitalisierungs-Schubs zu verstärken.

Aus einer bildungstheoretischen Perspektive kann weder der Ansatz ‘Selbsthilfe) noch die Idee einer (Entschulung) als unproblematisch wünschenswert angesehen werden. Einem potentiellen Machtgewinn non-formaler Strukturen durch Erklärvideos und Entschulungsprozesse als Auflösung problematischer Hegemonien steht in der faktischen Umsetzung doch häufig ein lediglich oberflächliches, selbstoptimiertes Lernen entgegen. Hier geht es um Zeitoptimierung, um Anpassung an ein hoheitlich verordnetes Lernen, dem man nicht entkommen kann, möglichst wenig störend für das eigene Leben zu machen. Kommerzielle Anbieter wie Simple Club haben dies erkannt und optimieren ihre Erklärvideos so, dass für die Schülerinnen und Schüler das gleiche Lernergebnis in Form der systemrelevanten Noten «schneller» zu erreichen ist, als in «langwierigen` Bildungsprozessen. Allerdings befördern die Schulen in ihrer aktuellen Ausformung dieses «Lernen ohne Bildungsanspruch» selbst und müssen sich den Spiegel der Erklärvideos und insbesondere der Tutorials vorhalten lassen - eine Optimierung des schulischen Lernens als eine effizienzorientierte Reduktion von schulischen Inhalten auf 7-Minuten Videos kann nur gelingen, wenn die Prüfungsprozesse dies zulassen.

Gleichzeitig zeigen weitere Daten in unserem Projekt, dass Schülerinnen und Schüler ausserhalb des schulbezogenen Lernens durchaus interessensgeleitet und verstehensorientiert mit Erklärvideos und Tutorials lernen (können). Wenn Schule dieses Potenzial von Erklärvideos und Tutorials aufgreifen würde, um primär nicht mehr nur zu vermitteln und zu prüfen, sondern zu unterstützen, zu fördern, Rückmeldungen zu geben und zu begleiten, könnte dies didaktische Wege zum 
selbstbestimmten Lernen öffnen, die ein vertieftes Lernen und eine hohe Expertisierung der kommenden Generation in einer sich dynamisch verändernden Gesellschaft ermöglichen (Wolf 2017). Chancenfördernde Qualifikation und Integration sowie eine diverse Enkulturation werden zukünftig für das Bildungssystem wichtiger sein als eine staatliche Allokation, welche nur zu Bildungsungerechtigkeiten führt.

\section{Literatur}

Birkelbach, Klaus, Rolf Dobischat, und Birte Dobischat. 2017. Ausserschulische Nachhilfe. Ein prosperierender Bildungsmarkt im Spannungsfeld zwischen kommerziellen und ??ffentlichen Interessen. Bd. 348. Study. Düsseldorf: Hans-Böckler-Stiftung. https://nbn-resolving. org/urn:nbn:de:101:1-2018072610440128611522.

Bourdieu, Pierre. 1983. «Ökonomisches Kapital, kulturelles Kapital, soziales Kapital». In Soziale Ungleichheiten, herausgegeben von Reinhard Kreckel, Sonderband 2:183-98. Soziale Welt. Göttingen: Schwartz.

Deursen, Alexander J. A. M. van, und Ellen J. Helsper. 2015. «The Third-Level Digital Divide: Who Benefits Most from Being Online?» In Studies in Media and Communications, herausgegeben von Laura Robinson, Shelia R. Cotten, Jeremy Schulz, Timothy M. Hale, und Apryl Williams, 10:29-52. Emerald Group Publishing Limited. https://doi.org/10.1108/S2050206020150000010002.

Dijk, Jan van. 2020. The digital divide. Cambridge, UK; Medford, MA: Polity.

Dohmen, Dieter, Annegret Erbes, Kathrin Fuchs, und Juliane Günzel. 2008. «Was wissen wir über Nachhilfe? Sachstand und Auswertung der Forschungsliteratur zu Angebot, Nachfrage und Wirkungen.» Köln: FIBS. https://tu-dresden.de/gsw/ew/ibbd/sp/ressourcen/dateien/forschung/online-archiv/FIBS.pdf.

Donker, Anouk S., Hester de Boer, Danny D. N. M. Kostons, Charlotte Dignath van Ewijk, und Margaretha P. C. Greetje van der Werf. 2014. «Effectiveness of Learning Strategy Instruction on Academic Performance: A Meta-Analysis». Educational Research Review 11 (Januar): 1-26. https://doi.org/10.1016/j.edurev.2013.11.002.

Feierabend, Sabine, Thomas Rathgeb, Hediye Kheredmand, und Stephan Glöckler. 2020. «JIMStudie 2019. Jugend, Information, Medien. Basisuntersuchung zum Medienumgang 12bis 19-Jähriger». Herausgegeben von Medienpädagogischer Forschungsverbund Südwest (mpfs). Medienpädagogischer Forschungsverbund Südwest (LFK, LMK). https://www.mpfs. de/fileadmin/files/Studien/JIM/2019/JIM_2019.pdf.

Feierabend, Sabine, Thomas Rathgeb, und Theresa Reutter. 2020. «JIM-Studie 2020. Jugend, Information, Medien. Basisuntersuchung zum Medienumgang 12- bis 19-Jähriger». Herausgegeben von Medienpädagogischer Forschungsverbund Südwest (mpfs). Medienpädagogischer Forschungsverbund Südwest (LFK, LMK). https://www.mpfs.de/fileadmin/files/ Studien/JIM/2020/JIM-Studie-2020_Web_final.pdf. 
Fend, Helmut. 2009. «Die sozialen und individuellen Funktionen von Bildungssystemen: Enkulturation, Qualifikation, Allokation und Integration». In Handbuch der Erziehungswissenschaft, herausgegeben von Christiane Hof, Thomas Fuhr, Wilhelm Wittenbruch, Stephanie Hellekamps, Wilfried Plöger, und Phillip Gonon, Band II: Teilband 1: Schule:43-55. Paderborn: Brill | Schöningh. https://doi.org/10.30965/9783657764969_005.

Giesecke, Alex, und Karsten D. Wolf. 2020. «Der SimpleClub: effiziente Vorbereitung auf die Klassenarbeit oder modernes Lernwerkzeug?» In Lehren und Lernen mit Tutorials und Erklärvideos: mit E-Book inside, herausgegeben von Stephan Dorgerloh und Karsten D. Wolf, 38-41. Pädagogik. Weinheim, Basel: Beltz.

Helmke, Andreas. 2021. Unterrichtsqualität und Lehrerprofessionalität: Diagnose, Evaluation und Verbesserung des Unterrichts. Aktualisierte 8. Auflage berücksichtigt die Hattie-Studien. Schule weiterentwickeln, Unterricht verbessern Orientierungsband. Hannover: Klett / Kallmeyer.

Herzig, Bardo. 2017. «Medien im Unterricht». In Lehrer-Schüler-Interaktion, herausgegeben von Martin K.W. Schweer, 503-22. Wiesbaden: Springer Fachmedien Wiesbaden. https://doi. org/10.1007/978-3-658-15083-9_22.

Illich, Ivan. 1971. Deschooling Society. World Perspectives Vol. 44. New York: Harper and Row.

Itō, Mizuko, Crystle Martin, Rachel Cody Pfister, Matthew H. Rafalow, Katie Salen, und Amanda Wortman. 2018. Affinity online: how connection and shared interest fuel learning. Connected Youth and Digital Futures. New York: New York University Press.

Jachmann, Michael. 2003. «Einstellung von Lehrern, Eltern und Schülern zur Leistungsbeurteilung - ein Vergleich». In Noten oder Berichte? Die schulische Beurteilungspraxis aus der Sicht von Schülern, Lehrern und Eltern, herausgegeben von Michael Jachmann, 201-27. Reihe Schule und Gesellschaft. Wiesbaden: VS Verlag für Sozialwissenschaften. https://doi. org/10.1007/978-3-322-97583-6_7.

Jürgens, Eiko. 2008. «Nachhilfeangebote». In Grundbegriffe Ganztagsbildung: Das Handbuch, herausgegeben von Thomas Coelen und Hans-Uwe Otto, 411-21. Wiesbaden: VS Verlag für Sozialwissenschaften. https://doi.org/10.1007/978-3-531-91161-8_41.

Kantereit, Tim, Hrsg. 2020. Hybrid-Unterricht 101. Visual Ink Publishing. https://visual-books. com/hybrid-unterricht-101.

Klemm, Klaus, und Nicole Hollenbach-Biele. 2016. «Nachhilfeunterricht in Deutschland. Ausmaß - Wirkung - Kosten.» Gütersloh: Bertelsmann Stiftung. https://www.bertelsmann-stiftung.de/fileadmin/files/BSt/Publikationen/GrauePublikationen/Nachhilfeunterricht_in_ Deutschland_160127.pdf.

Kommer, Sven. 2010. Kompetenter Medienumgang?: Eine qualitative Untersuchung zum medialen Habitus und zur Medienkompetenz von SchülerInnen und Lehramtsstudierenden. Opladen: Verlag Barbara Budrich. https://www.jstor.org/stable/j.ctvbkjtnp.

Kommer, Sven. 2016. "Buch statt Tablet-PC : Warum die digitalen Medien nicht in die Schule Kommen - der Faktor LehrerIn». In Wi(e)derstände : digitaler Wandel in Bildungseinrichtungen, herausgegeben von Thomas Knaus und Olga Engel, 5:35-68. fraMediale. München: kopaed. 
Lévi-Strauss, Claude. 1973. Das wilde Denken. Frankfurt am Main: Suhrkamp.

Müller, Christian. 2012. «Warum Rational Choice?» In Ökonomik als allgemeine Theorie menschlichen Verhaltens, herausgegeben von Christian Müller, Frank Trosky, und Marion Weber, 3-20. De Gruyter. https://doi.org/10.1515/9783110508024-002.

Rat für Kulturelle Bildung, Hrsg. 2019. Jugend/Youtube/Kulturelle Bildung - Horizont 2019 Studie: eine repräsentative Umfrage unter 12- bis 19-jährigen zur Nutzung kultureller Bildungsangebote an digitalen Kulturorten. Essen: Rat für Kulturelle Bildung e. V. https://www.ratkulturelle-bildung.de/fileadmin/user_upload/pdf/Studie_YouTube_Webversion_final.pdf.

Rathgeb, Thomas. 2020. «JIMplus 2020. Lernen und Freizeit in der Corona-Krise». Medienpädagogischer Forschungsverbund Südwest (LFK, LMK). https://www.mpfs.de/fileadmin/files/ Studien/JIM/JIMplus_2020/JIMplus_2020_Corona.pdf.

Rheinische Post. 2020. «Karliczek: Schulen müssen nach den Ferien volles Programm anbieten - notfalls mit Containern als Klassenräumen». Pressemeldung. presseportal.de. 30. Mai 2020. https://www.presseportal.de/pm/30621/4610096.

Ricken, Norbert. 2021. "Optimierung - eine Topographie». In Optimierung. Anschlüsse an den 27. Kongress der Deutschen Gesellschaft für Erziehungswissenschaft, herausgegeben von Henrike Terhart, Sandra Hofhues, und Elke Kleinau, 66:21-44. Schriften der Deutschen Gesellschaft für Erziehungswissenschaft (DGFE). Opladen: Verlag Barbara Budrich. https:// doi.org/10.3224/84742485.

Ryan, Richard M, und Edward L. Deci, Hrsg. 2017. Self-Determination Theory: Basic Psychological Needs in Motivation, Development, and Wellness. New York: Guilford Press. https://doi. org/10.1521/978.14625/28806.

Seele, Peter. 2020. «Gefährden Googles Abschlüsse die Hochschulen?» Forschung \& Lehre, 22. Oktober 2020, Abschn. Zeitfragen. https://www.forschung-und-lehre.de/zeitfragen/gefaehrden-googles-abschluesse-die-hochschulen-3173/.

STARK-Verlag. O. J. «STARK Buchhandels-Partnerprogramm». Pearson Deutschland GmbH / STARK Verlag GmbH. https://www.stark-verlag.de/buchhandel/partner.

Studienkreis. 2019. «Studienkreis-Nachhilfe: Zahlen \& Fakten». 2019. https://www.studienkreis.de/unternehmen/presse/zahlen-und-fakten/studienkreis-zahlenmaterial/.

Verständig, Dan, Alexandra Klein, und Stefan Iske. 2016. «Zero-Level Digital Divide. Neues Netz und neue Ungleichheiten». SIEGEN:SOZIAL - Analysen, Berichte, Kontroversen (SI:SO) 21: 50-55. https://nbn-resolving.org/urn:nbn:de:hbz:467-11973.

Wagner, Petra, Barbara Schober, und Christiane Spiel. 2005. «Wer hilft beim Lernen für die Schule?» Zeitschrift für Entwicklungspsychologie und Pädagogische Psychologie 37 (2): 101-9. https://doi.org/10.1026/0049-8637.37.2.101.

Wild, Klaus-Peter, und Ulrich Schiefele. 1994. «Lernstrategien im Studium: Ergebnisse zur Faktorenstruktur und Reliabilität eines neuen Fragebogens». Zeitschrift für Differentielle und Diagnostische Psychologie 15 (4): 185-200.

Wolf, Karsten D. 2015a. «Bildungspotenziale von Erklärvideos und Tutorials auf YouTube. Audiovisuelle Enzyklopädie, adressatengerechtes Bildungsfernsehen, Lehr-Lern-Strategie oder partizipative Peer Education?» merz - medien + erziehung 1: 30-36. 
Wolf, Karsten D. 2015b. «Produzieren Jugendliche und junge Erwachsene ihr eigenes Bildungsfernsehen? Erklärvideos auf YouTube». TelevIZIon 28 (1): 35-39. http://www.br-online.de/ jugend/izi/deutsch/publikation/televizion/28_2015-1/Wolf-Produzieren_Jugendliche_ und_junge_Erwachsene.pdf.

Wolf, Karsten D. 2017. «Mediatisierung in reformpädagogischen Lernkulturen». In Handbuch Reformpädagogik, herausgegeben von Till-Sebastian Idel und Heiner Ullrich, 338-52. Beltz-Handbuch. Weinheim: Beltz.

Wolf, Karsten D., Ricarda Bolten, und Konstanze Wegmann. 2019. «Kurzfragebogen „Lernstrategien bei der Nutzung von Erklärvideos und Tutorials“». Bremen: ZeMKI Lab Medienbildung \& Bildungsmedien. https://blogs.uni-bremen.de/bildungslab/files/LeSNET-Kurz-2019.pdf.

Wolf, Karsten D., und Christoph Kulgemeyer. 2021. "Lehren und Lernen mit Erklärvideos im Fachunterricht». In Handbuch Lernen mit digitalen Medien, herausgegeben von Gerold Brägger und Hans-Günter Rolff, 472-85. Reihe Pädagogik. Weinheim, Basel: Beltz.

Ziegler, Albert, Kimberley L. Chandler, Wilma Vialle, und Heidrun Stoeger. 2017. «Exogenous and Endogenous Learning Resources in the Actiotope Model of Giftedness and Its Significance for Gifted Education». Journal for the Education of the Gifted 40 (4): 310-33. https:// doi.org/10.1177/0162353217734376.

Ziegler, Albert, Manuel Hopp, Markus Linner, Tanja Schöferle, Niklas Thomas, Tanja Schielein, Anamaria Vladut, und Wolfgang Götzfried. 2012. «Bildungs- und Lernkapitalarmut von Hauptschülern: Ergebnisse einer explorativen Befragungsstudie mit Lehrkräften». Schulpädagogik-heute. http://psycho.ewf.uni-erlangen.de/mitarbeiter/ziegler/publikationen/ Publikation17a.pdf.

Ziegler, Albert, Wilma Vialle, und Bastian Wimmer. 2013. "The Actiotope Model of Giftedness: An Introduction to Some Central Theoretical Assumptions». In Exceptionality in East Asia. Explorations in the Actiotope Model of Giftedness, herausgegeben von Shane N. Phillipson, Heidrun Stoeger, und Albert Ziegler, 1-17. London: Routledge. https://doi. org/10.4324/9780203126387.

Zwingenberger, Anja. 2009. Wirksamkeit multimedialer Lernmaterialien. Bd. 75. Pädagogische Psychologie und Entwicklungspsychologie. Münster: Waxmann.

Zydorek, Christoph. 2017. «Einige ökonomische Grundbegriffe (1): Menschliches Verhalten». In Einführung in die Medienwirtschaftslehre, herausgegeben von Christoph Zydorek, 33-45. Wiesbaden: Springer Fachmedien. https://doi.org/10.1007/978-3-658-14217-9_4.

\section{Förderangaben}

Das Projekt wurde gefördert durch das BMBF (FKZ 01JD1804). Zusätzliche Datenerhebungen und Analysen wurden durch die Stiftung Mercartor gefördert.

\section{Danksagung}

Wir danken den Gutachtenden für wertvolle Hinweise zur Überarbeitung des Manuskriptes. 